\title{
The State of the Evidence for Whole-System, Multi-Modality Naturopathic Medicine: A Systematic Scoping Review
}

\author{
Stephen P. Myers, PhD, BMed, ND, ${ }^{1-3}$ and Vanessa Vigar, BNat (Hons) ${ }^{1,2,4}$
}

\section{Abstract}

Objective: To summarize the current state of the research evidence for whole-system, multi-modality naturopathic medicine.

Design: A systematic search for research articles from around the world was undertaken using MEDLINE, Embase, CINAHL, AMED, and WHO regional indexes. Naturopathic journals and gray literature were hand searched. No language restrictions were imposed.

Interventions: All human research evaluating the effectiveness of naturopathic medicine, where two or more naturopathic modalities are delivered by naturopathic clinicians, were included in the review. Case studies of five or more cases were included.

Results: Thirty-three published studies $(n=9859)$ met inclusion criteria (11 American; 4 Canadian; 6 German; 7 Indian; 3 Australian; 1 United Kingdom; and 1 Japanese) across a range of mainly chronic clinical conditions. The studies predominantly showed evidence for the efficacy of naturopathic medicine for the conditions and settings in which they were based.

Conclusions: To date, research in whole-system, multi-modality naturopathic medicine shows that it is effective for treating cardiovascular disease, musculoskeletal pain, type 2 diabetes, polycystic ovary syndrome, depression, anxiety, and a range of complex chronic conditions.

Keywords: naturopathic medicine, naturopathy, global, systematic review, whole-system, pragmatic

\section{Introduction}

$\mathbf{N}$ ATUROPATHIC MEDICINE IS AN eclectic practice of health care united by core underlying philosophy, theory, and principles. A central tenet of naturopathic philosophy is vis medicatrix naturae (the healing power of nature), an ancient concept often ascribed to Hippocrates, ${ }^{1,2}$ that refers to an inherent, self-organizing healing process in living systems which establishes, maintains, and restores health. ${ }^{1}$

The terms "whole-system" \& "multi-modality" within the context of naturopathic medicine are outlined in Table 1. Broadly these refer to the practice of naturopathic medicine as a complex health care intervention, which utilizes a combination of clinical modalities (or therapies) in the treatment of each individual. This contrasts with a single modality approach where only one therapy is used. Modalities used in naturopathic practice are determined by a structured system of theory and principles based within its philosophy; refer to Table 1 for a list of core modalities.

The World Health Organization (WHO) defines naturopathy as part of Traditional and Complementary Medicine (T\&CM) and has recommended this sector to build evidence to support its safe and effective use. ${ }^{3}$ The imperative to increase the evidence base of T\&CM results from the emergence of evidence-based medicine (EBM) in the last quarter of the twentieth century. ${ }^{4}$ While a substantial body of evidence for the effectiveness of the "tools of trade" of naturopathic medicine (i.e., herbal and nutritional supplements;

\footnotetext{
${ }^{1}$ National Centre for Naturopathic Medicine, Southern Cross University, Lismore, Australia.

${ }^{2}$ NatMed Research Unit, Division of Research, Southern Cross University, Lismore, Australia.

${ }^{3}$ Foundations of Naturopathic Medicine Institute, Snoqualmie, Washington.

${ }^{4}$ Integria Healthcare, Ballina, Australia.
}

(C) Stephen P. Myers and Vanessa Vigar, 2019; Published by Mary Ann Liebert, Inc. This Open Access article is distributed under the terms of the Creative Commons License (http://creativecommons.org/licenses/by/4.0), which permits unrestricted use, distribution, and reproduction in any medium, provided the original work is properly cited. 
Table 1. Definitions for Key Study Components

\section{Naturopathic medicine}

Multi-modality

Whole system

The WNF defines the naturopathic (healing power of nature; treat the no harm; doctor as teacher; health wellness). ${ }^{10}$ The philosophy, theory, modalities. The WNF has identified seven core modalities: (1) clinical nutrition and diet modification/ dietary supplements, traditional medicines, and natural health care products); (3) herbal medicine; (4) (6) homeopathy, including complex homeopathy; and (7) physical modalities (based on the treatment jurisdiction, including yoga, release techniques). ${ }^{10}$ This scoping study is limited to naturopathic by the WNF. Other systems of traditional medicine, such as Traditional Chinese Medicine and Ayurveda, are not included in this systematic scoping review study.

Within the context of this systematic scoping review, "multi-modality be more indicative of that specific modality, rather than eclectic naturopathic general practice.

Refers to the practice of naturopathic profession based on two fundamental philosophies of medicine (vitalism and holism) and seven principles of practice whole person; treat the cause; first, do promotion and disease prevention; and and principles are translated to clinical practice through a range of therapeutic counseling; (2) applied nutrition (use of lifestyle counseling; (5) hydrotherapy; modalities taught and allowed in each naturopathic manipulation, and muscle medicine as defined and encompassed naturopathic practice" was defined as including a minimum of two modalities as part of a single clinical approach to treatment of an individual. The practice of a single modality was considered to medicine as a complex health care intervention that addresses simultaneously the multiple dimensions (physical, mental, spiritual, family, community, and environment) of an individual patient ${ }^{1}$ as pragmatically practiced by naturopathic clinicians.

WNF, World Naturopathic Federation.

and lifestyle interventions [LIs]) is now available, there exists little quantitative scientific evidence documenting it as an effective medical practice. ${ }^{5}$

The movement toward developing a scientific evidence base for naturopathic medicine is not without controversy. Some have argued that EBM is antithetical to naturopathy, out of concern that traditional naturopathic philosophy and practice will be marginalized or excluded in a process of coercing nonorthodox systems of health and healing to fit into the mainstream scientific paradigm. ${ }^{6}$ Others argue that although there exist inevitable tensions between T\&CM and
EBM epistemologies, these tensions and their resolutions also can hold the key to a more productive understanding between traditional and scientific knowledge. ${ }^{7}$

Galvanized by this need to develop a body of quantitative scientific evidence supporting naturopathic medicine, a group of U.S. naturopathic researchers received a grant from the National Institutes of Health's National Center for Complementary and Integrative Health (previously the National Center for Complementary and Alternative Medicine) in 2006 to develop a Naturopathic Medical Research Agenda (NMRA). ${ }^{8}$ The project involved research directors from every North American institution with a naturopathic program, Southern Cross University (an Australian institution with a publicly funded naturopathic medicine program), along with 1200 naturopathic academics, practitioners, students, and selected medical researchers. The primary recommendation from the NMRA was that research be conducted on the whole practice of naturopathic medicine, rather than on single agents (such as individual herbal or nutritional supplements). ${ }^{8}$

Following the NMRA recommendations, significant research occurred, especially in North America. In 2015, a systematic review of this research, ${ }^{9}$ including 15 clinical studies reporting on the outcomes of multi-modality treatment delivered by North American naturopathic physicians, was published. They concluded that while many sample sizes were small, results indicated that receiving wholesystem naturopathic medicine was associated with improved health outcomes and improved quality of life (QOL) in patients with or at risk for chronic conditions, including cardiovascular disease (CVD), type 2 diabetes, chronic pain, anxiety, multiple sclerosis, hepatitis $\mathrm{C}$, and menopausal symptoms. ${ }^{9}$ Also in $2015,{ }^{9}$ the World Naturopathic Federation (WNF) convened its inaugural meeting. The WNF now represents more than 50 international naturopathic organizations with a primary goal to promote and advance the naturopathic profession. Given this international interest, it is timely to undertake a systematic scoping review that summarizes the state of the evidence for whole-system, multi-modality naturopathic medicine across the world. A systematic scoping review differs from a systematic review in that it sets out to examine the extent, range, and nature of research activity in a broad area, ${ }^{10}$ while a systematic review generally sets out to answer a focused question by synthesizing all available research. The main goal of this scoping study is to highlight the breadth of the quantitative scientific research in naturopathic medicine.

\section{Methods}

In July 2018, the authors undertook a comprehensive search of MEDLINE, Embase, CINAHL, AMED, and the WHO regional indexes (AIM, LILACS, IMEMR, IMSEAR, WPRIM). The MEDLINE search strategy is shown in Figure 1; other search strings are available upon request.

In addition, the authors conducted additional hand searches of the following journals: British Naturopathic Journal, Townsend Letters, Journal of the Australian Traditional Medicine Society, The International Journal of Naturopathic Medicine, and the Journal of Orthomolecular Medicine. Submissions to the Australian Natural Therapy Review regarding the effectiveness of naturopathic medicine ${ }^{11}$ were also searched for additional references. 
\#1 Naturopathy [mh] OR naturopath* [tw]

\#2 Therapeutics [mh] OR therapy [sh] OR "therapeutic use" [sh] OR treatment* [tw] OR

therapy [tw] OR therapies [tw] OR intervention* [tw] OR multimodal* [tw] OR multi-modal*

[tw] OR modalit* [tw] OR "whole practice" [tw] OR "whole system" [tw] OR "whole

systems" [tw] OR Quality of Life [mh] OR life qualities [tw] OR life quality [tw] OR quality

adjusted [tw] OR adjusted life [tw]

FIG. 1. Medline Search

\#3 Patients [mh] OR patient [tw] OR client [tw] OR clients [tw] OR consumer [tw] OR

consumers [tw] OR participant [tw] OR Treatment Outcome [mh] OR Program Evaluation

[mh] OR outcome ${ }^{*}[\mathrm{tw}]$ OR evaluat* $[\mathrm{tw}]$ OR impact* $[\mathrm{tw}]$ OR effective* $[\mathrm{tw}]$ OR efficac*

[tw] OR qualit* [tw]

\#4 1 AND 2 AND 3

Studies were included if they met the following criteria:

(1) Controlled clinical trials, longitudinal cohort studies, observational trials, or case series involving five or more cases presented in any language

(2) Human studies

(3) Multi-modality treatment administered by a naturopath (naturopathic clinician, naturopathic physician) as an intervention

(4) Non-English language studies in which an English title and abstract provided sufficient information to determine effectiveness

(5) Case series in which five or more individual cases were pooled and authors provided a summative discussion of the cases in the context of naturopathic medicine

It was decided that case series with less than five cases were more representative of the individual cases, rather than an evaluation of whole-system naturopathic treatment in a specific condition.

Titles and abstracts were screened by both authors, based on the inclusion criteria, with disagreements settled by discussion.

See Figure 2 for a flowchart documenting the study selection.

\section{Data extraction}

The following data from included studies were extracted (V.V.) and summarized using a data extraction sheet: study reference, origin, condition, number of participants, study design, primary outcomes, patient-reported outcomes, and modalities utilized. Data were divided by conditions treated (Tables 2-8).

\section{Methodological assessment and quality rating}

Risk of bias assessment was conducted on all clinical trial reports using the Cochrane Collaboration Risk of Bias tool (Fig. 3). ${ }^{12}$

\section{Results}

\section{Studies meeting inclusion criteria}

A total of 2551 titles were located and the titles and abstracts screened for eligibility. Full text of 95 primary studies and 12 gray literature studies were reviewed in detail (totalling 107). Thirty-three articles were accepted for inclusion, totalling 9859 study participants. The primary reasons for exclusion were lack of multi-modality treatment (i.e., only a single modality was practiced) or the studies did not identify that treatments were conducted by naturopathic clinicians. Notably, studies were excluded because they prescribed naturopathic-style treatments, but were administered by integrative doctors or other personnel not identified as naturopathic. Two poster presentations were included, ${ }^{13,14}$ in which the data were relevant and not reported elsewhere.

\section{Characteristics of included studies}

A majority of the included studies were observational cohort studies (12 prospective and 8 retrospective), with 11 clinical trials and 2 case series (Table 9). Studies were reported from seven different locations: United States (US), Canada, Germany, India, Australia, Japan, and the United Kingdom. The studies are diverse in nature, representing short-term inpatient care (primarily in studies from India and Germany) to longer term observational reports of outpatient treatment (primarily in the US and Canada). The settings of care included private naturopathic practice, integrative hospitals, inpatient health care clinics, or research institutes.

\section{Research locations}

United States. Eleven articles from the US are included in this review. Of these, nine also are included in the Oberg et al. (2015) systematic review of naturopathic whole system practice in North America. ${ }^{9}$ Two additional studies 


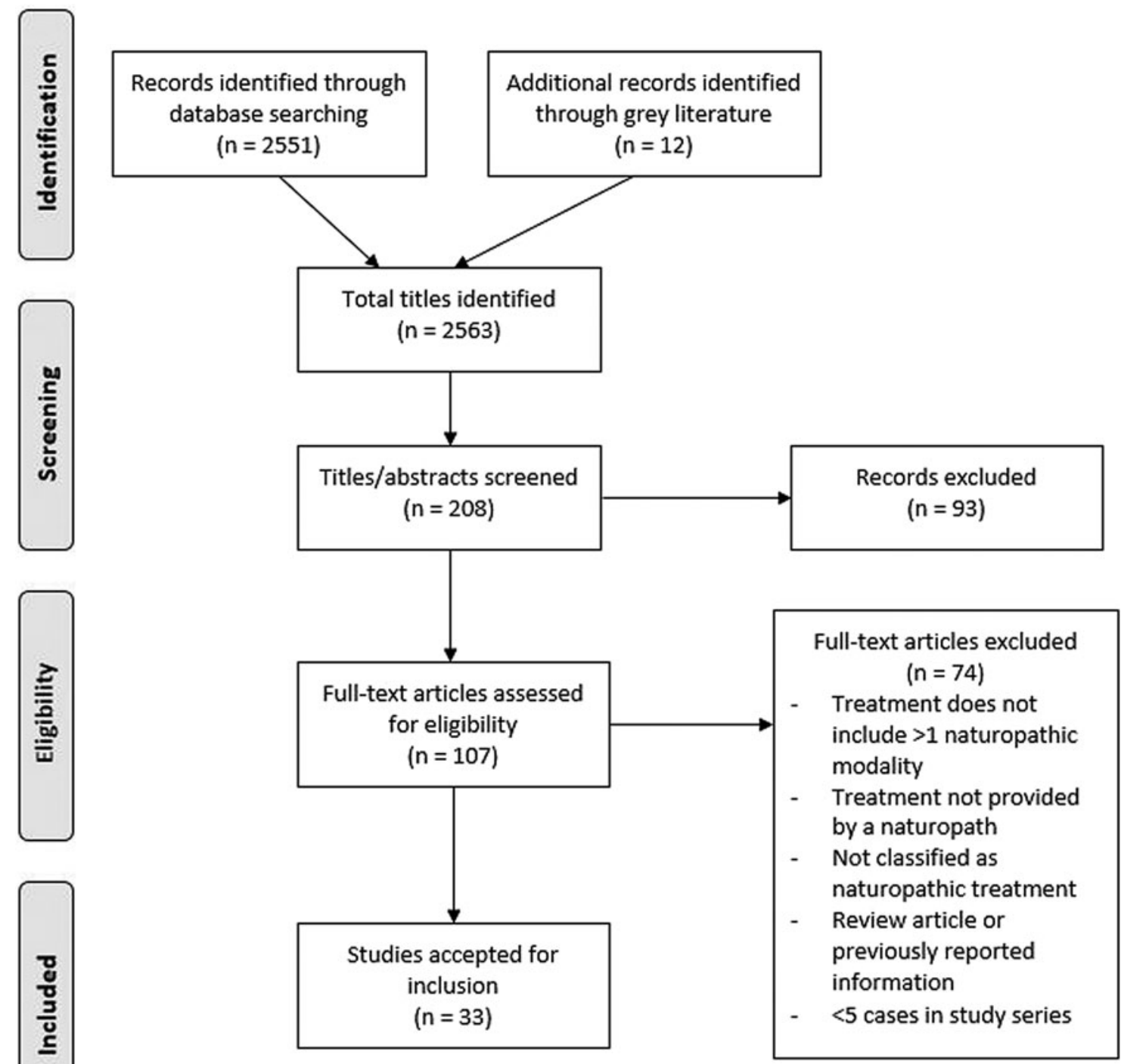

FIG. 2. Flow diagram of study selection.

published after the 2015 review also were identified. ${ }^{15,16}$ All studies were of chronic disease conditions: three in chronic pain management of different etiologies ${ }^{17-19}$; three evaluated outcomes in type 2 diabetes management ${ }^{20-22}$; two in mood disorders ${ }^{15,16}$; and one on treatment of hypertension. ${ }^{23}$ The remaining two studies included treatment for hepatitis $\mathrm{C}^{24}$ and menopausal symptoms. ${ }^{25}$

Seven publications were prospective or retrospective observational studies, ${ }^{15,20-25}$ three were randomized controlled trials (RCTs), ${ }^{17-19}$ and one was a case series of difficult-to-treat patients. ${ }^{16}$ All the studies were conducted in the community in either public or private naturopathic clinics or in community health centers.

The naturopathic modalities included diet counseling and physical activity recommendations, stress reduction strategies, dietary supplements, hydrotherapy, manual therapy, and botanical medicines. ${ }^{9}$
Canada. Four articles were conducted in Canada specifically in a population of Canada Post employees. These included two in chronic pain management, ${ }^{26,27}$ one in anxiety, ${ }^{28}$ and one for primary prevention in CVD. ${ }^{29}$ All of these studies were RCTs, conducted at work-site clinics, and all were included in the Oberg et al. review of 2015. Naturopathic modalities included dietary counseling, nutritional supplementation, and relaxation techniques. One study used acupuncture in addition to the other modalities. ${ }^{27}$

Germany. Six articles from Germany were identified for inclusion. ${ }^{14,30-34}$ Two were conducted in the treatment of musculoskeletal pain, ${ }^{14,33}$ three in various chronic conditions of mixed etiology, ${ }^{31,32,34}$ and one examining QOL measures. ${ }^{35}$ Five were conducted in integrative hospitals, and one was conducted in a residential, long-term care setting. The naturopathic care reported in these studies is from integrative inpatient 


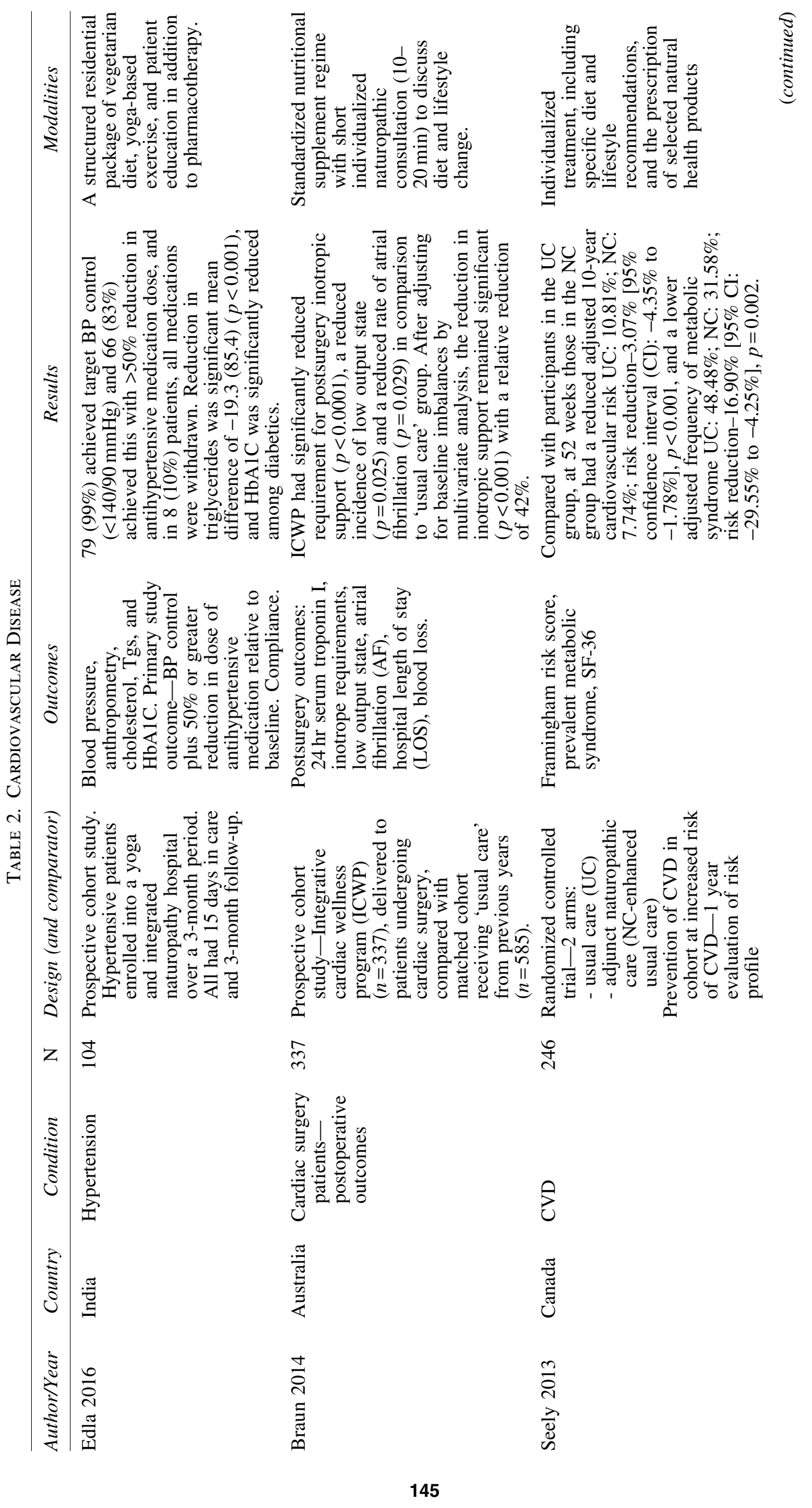




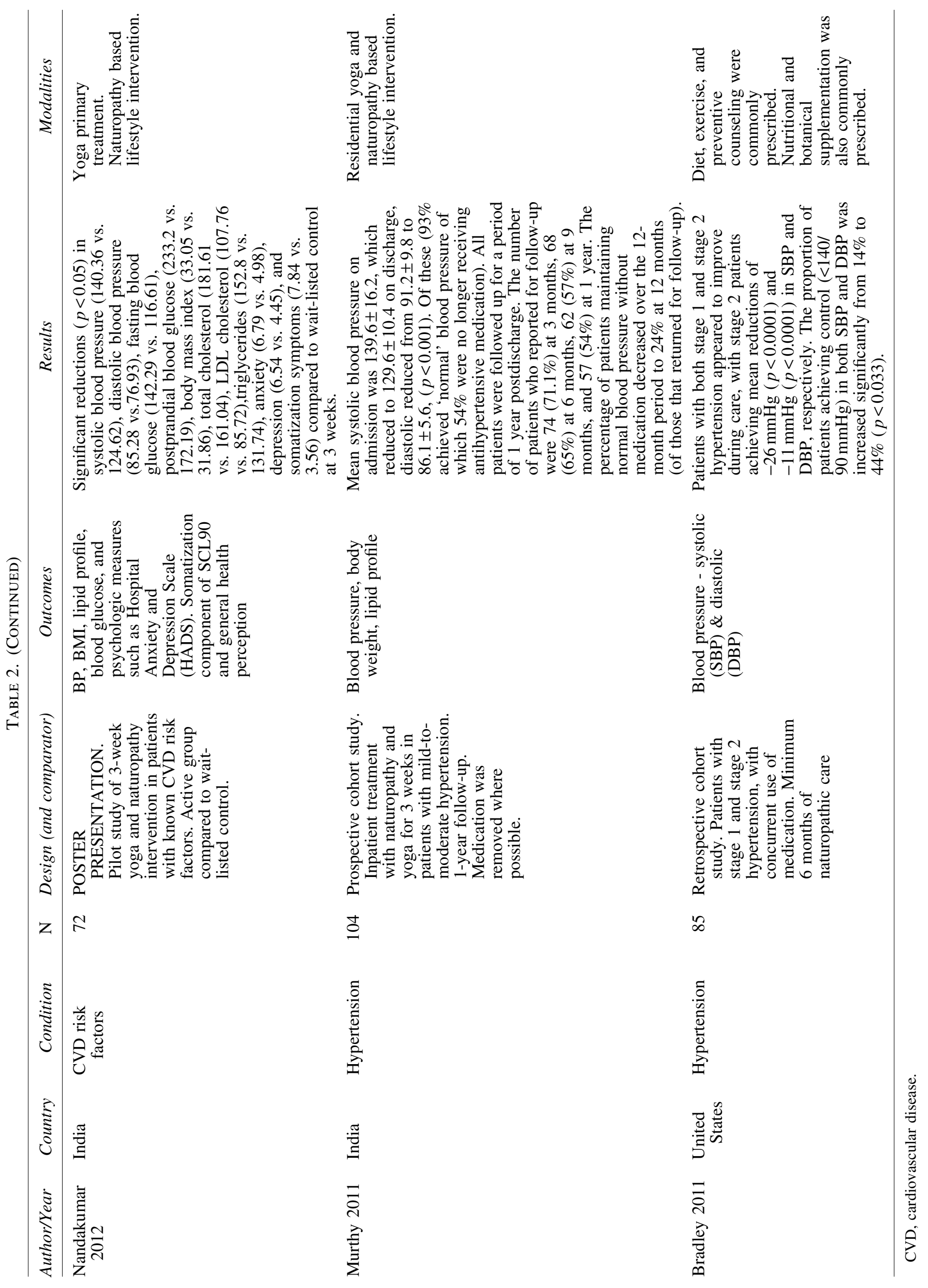




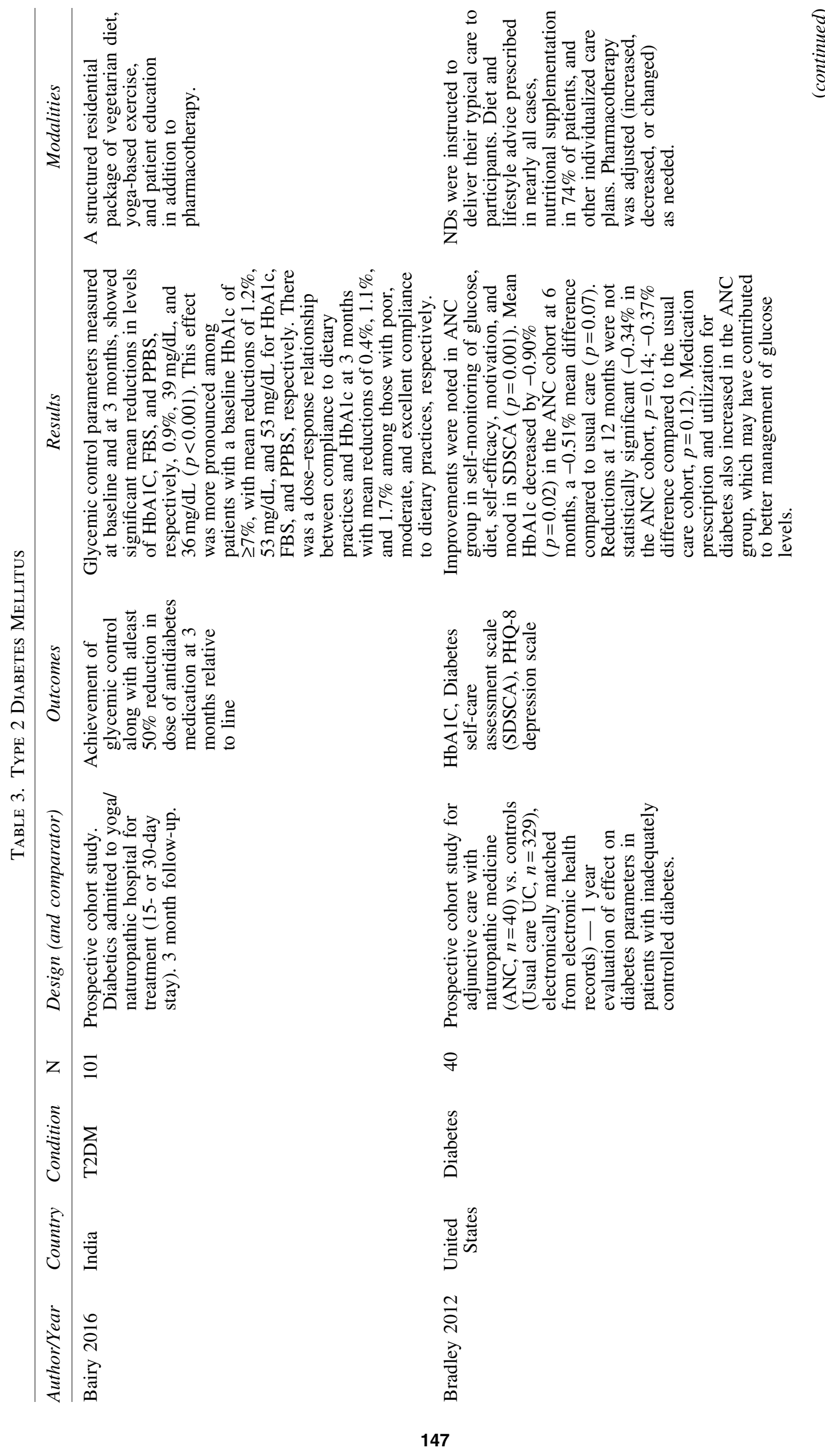




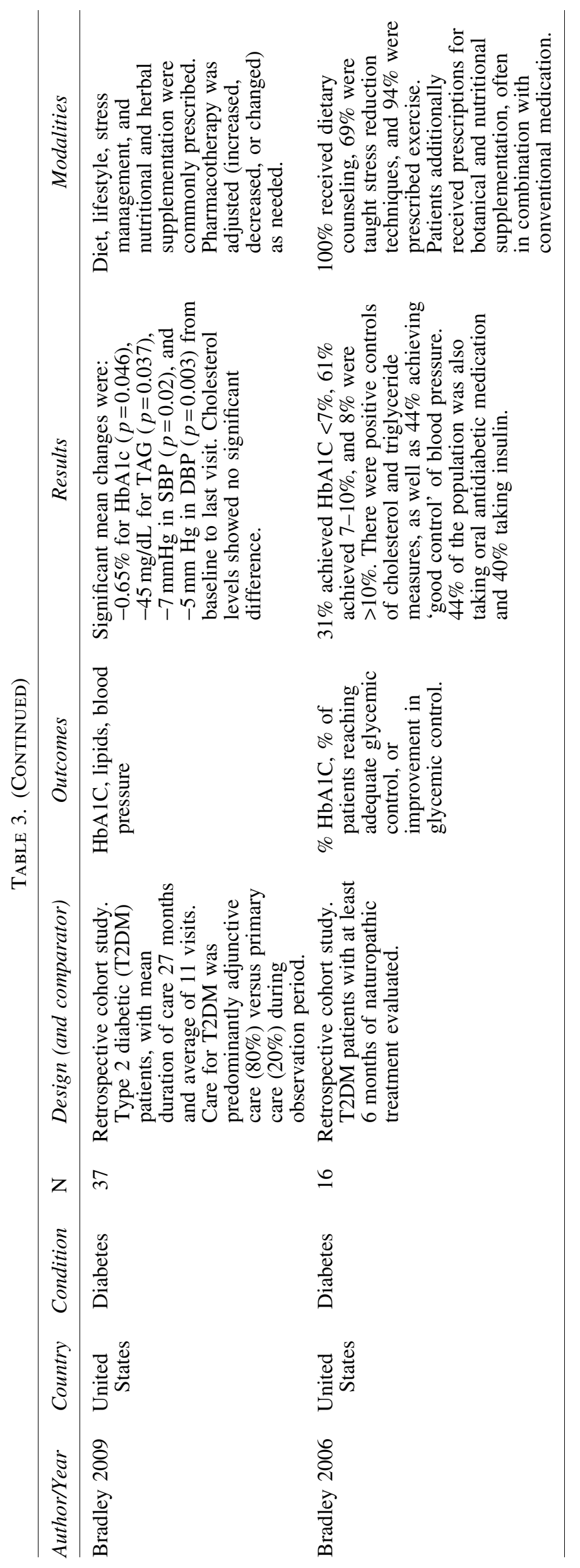




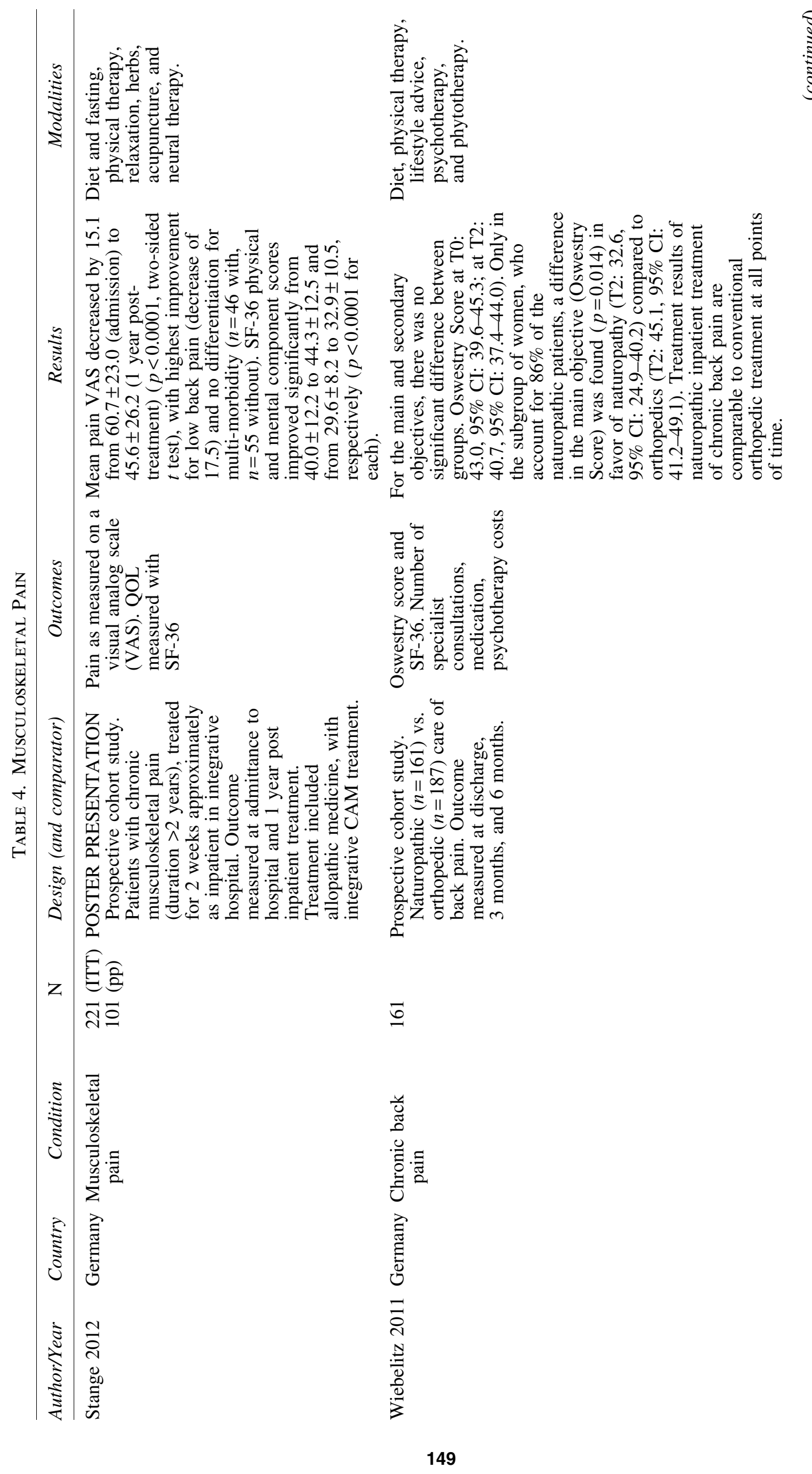




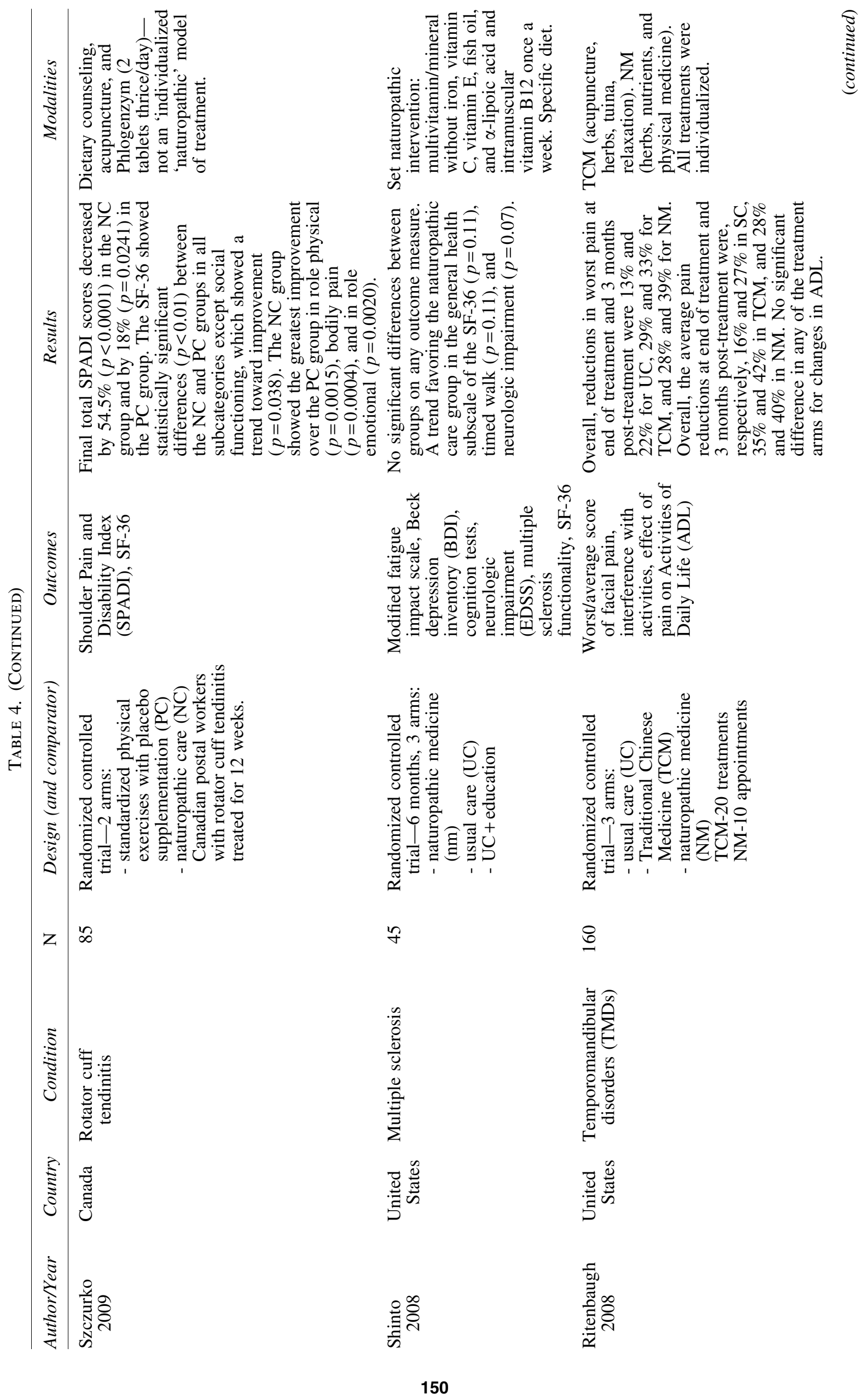




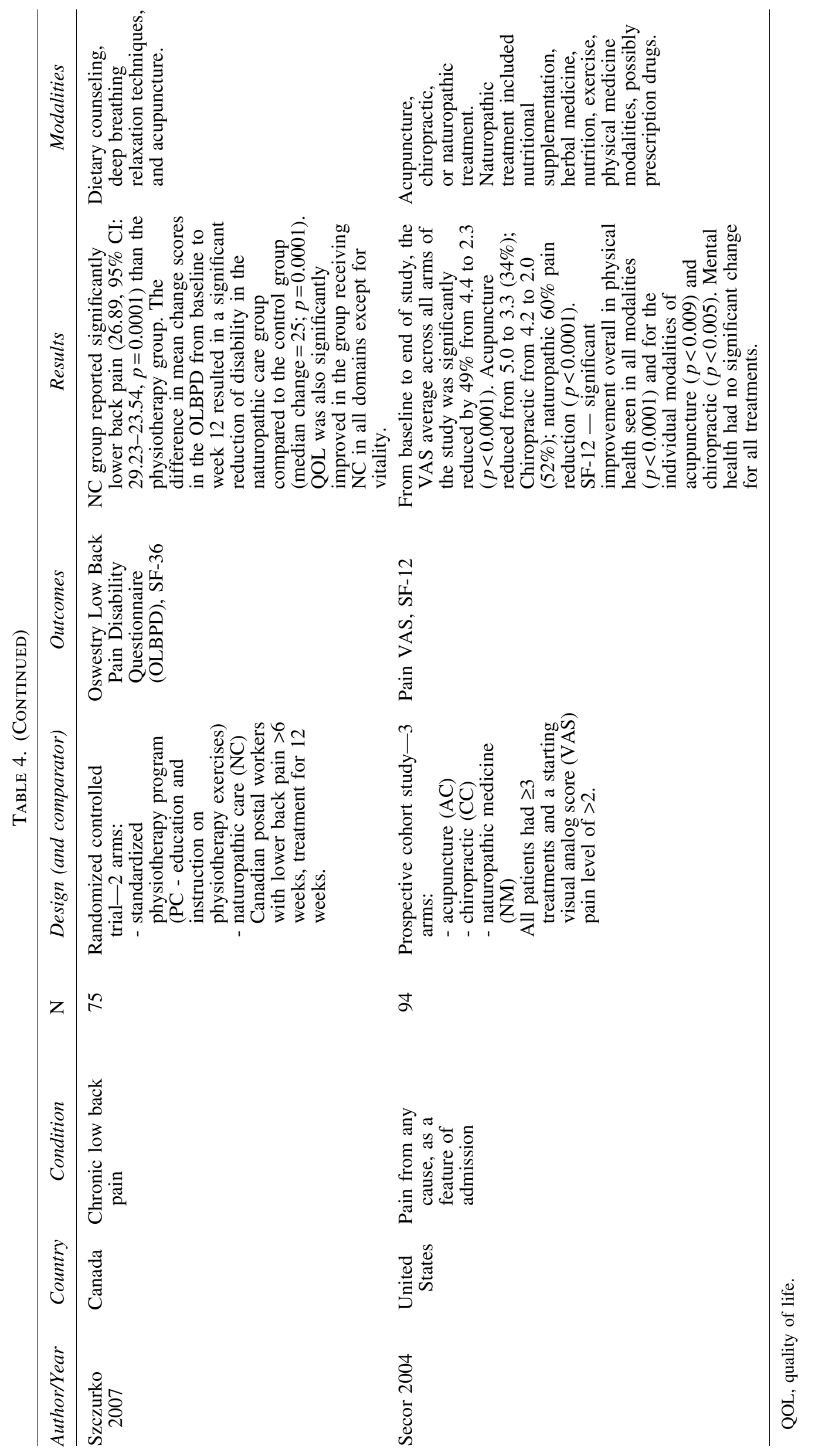




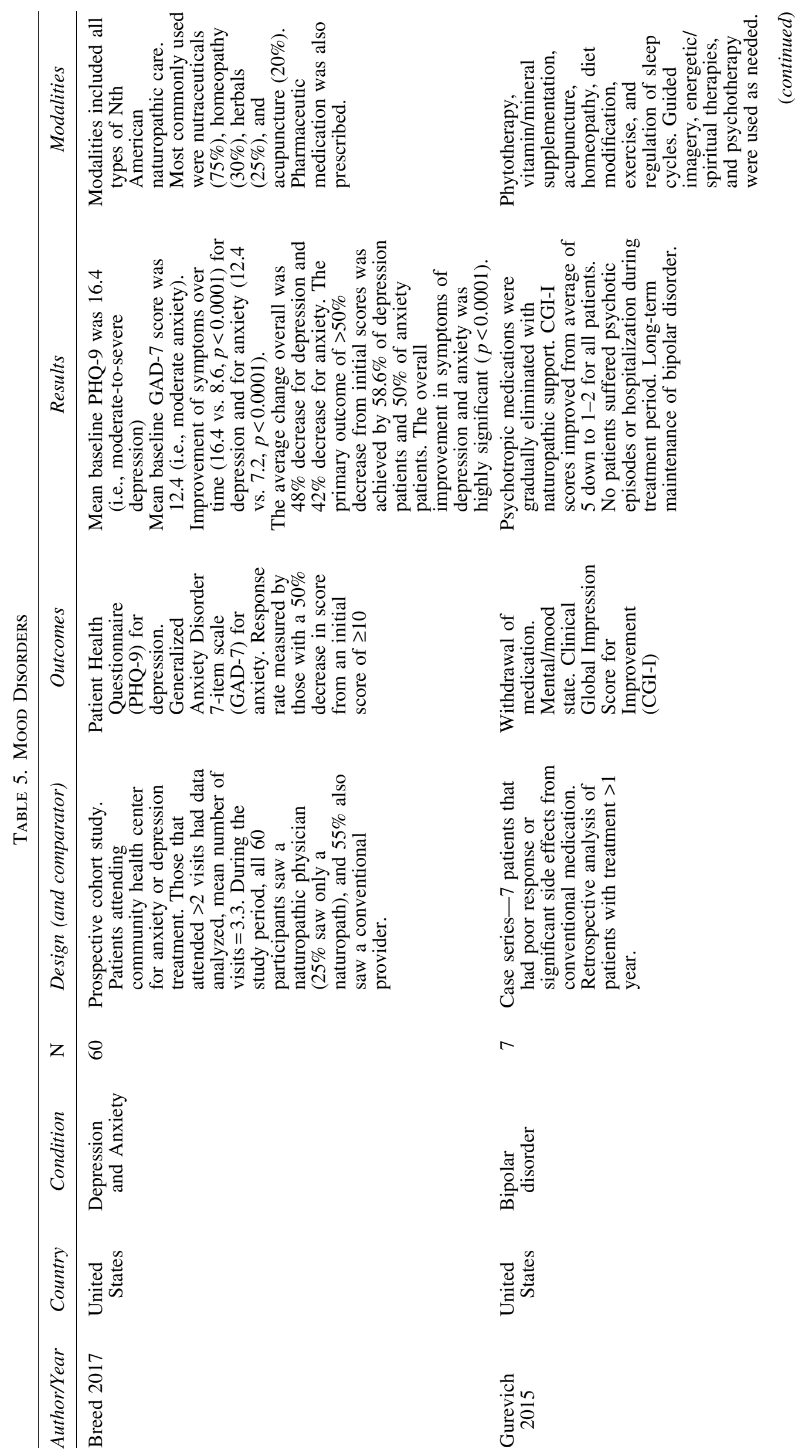




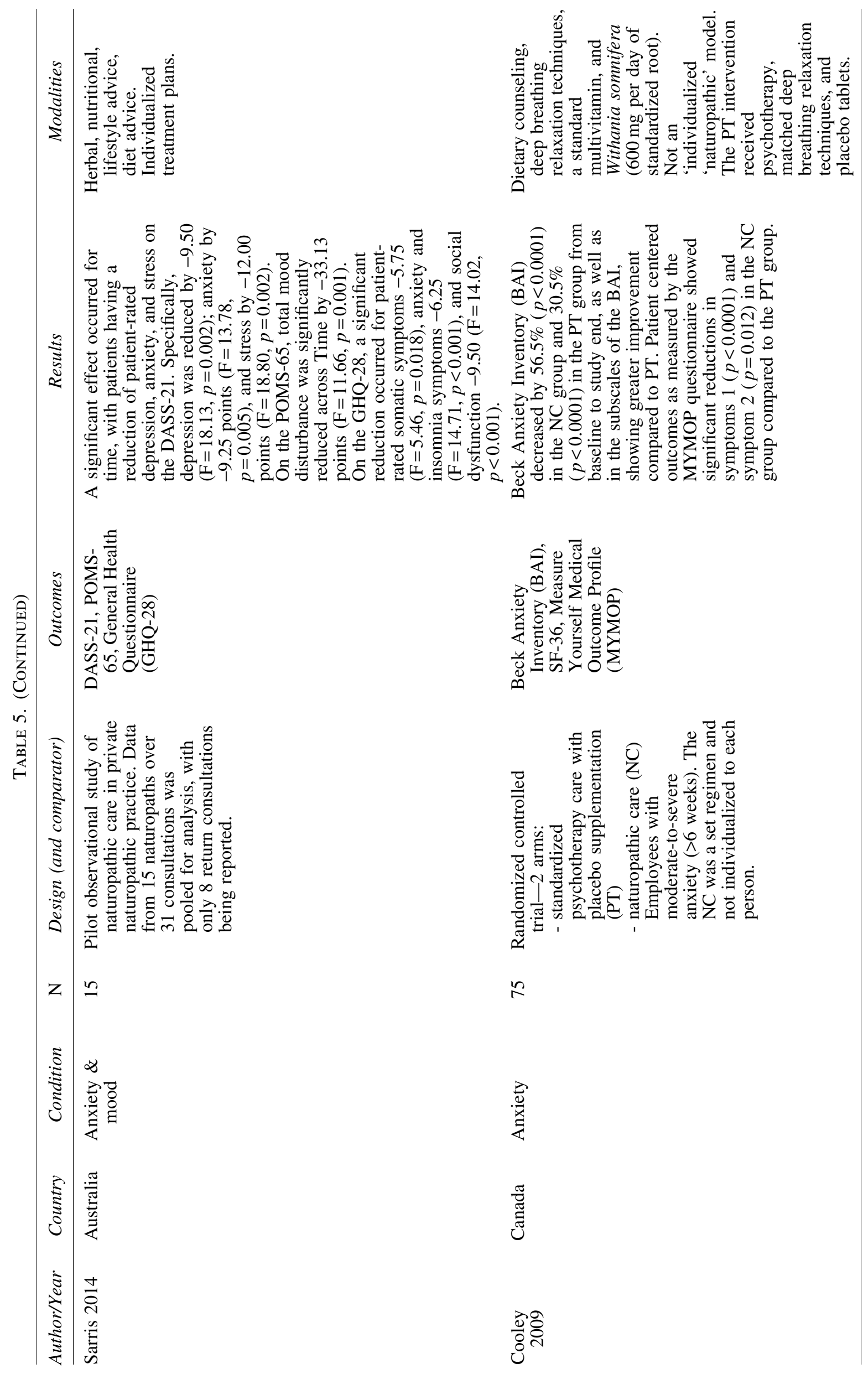




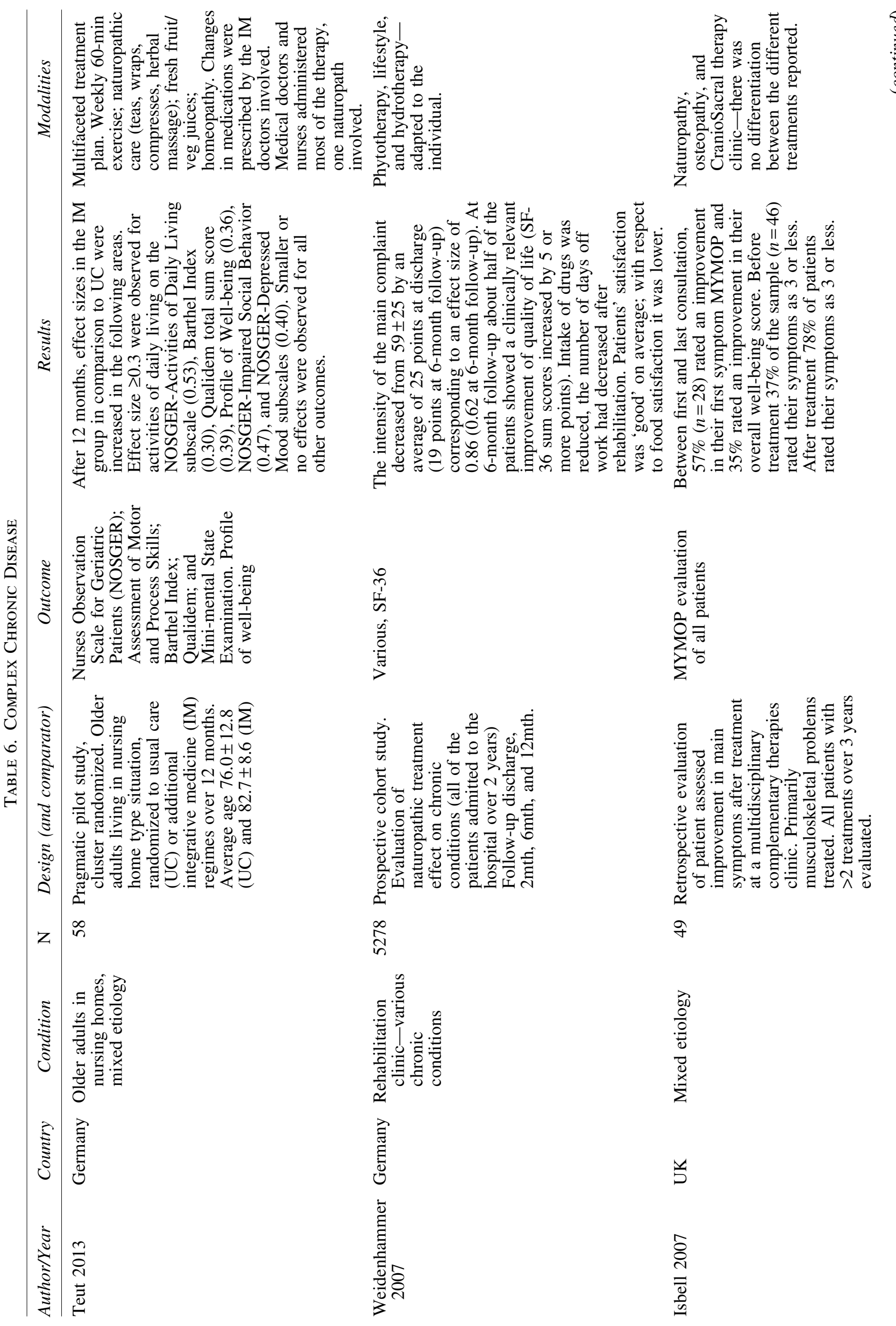




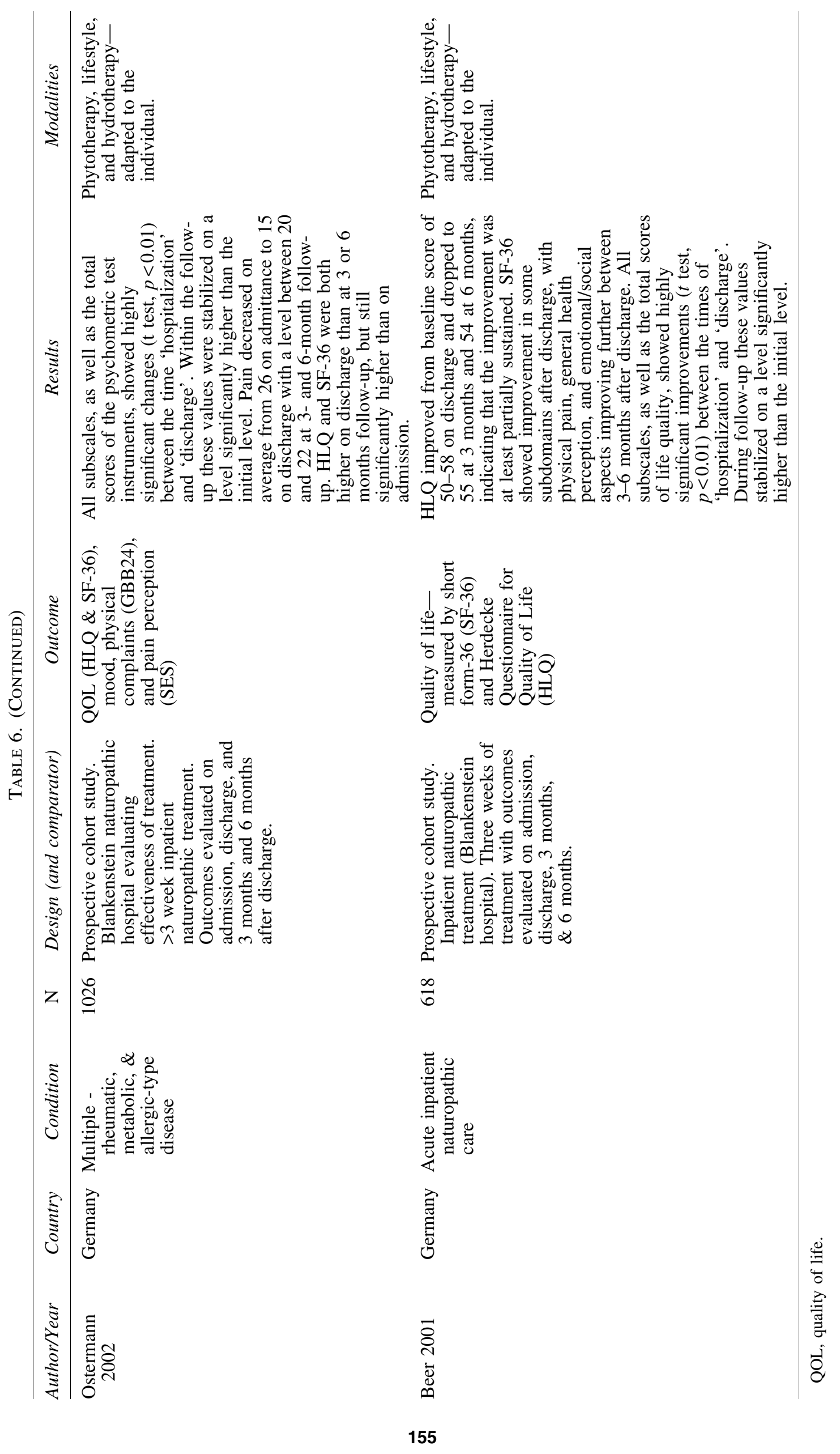




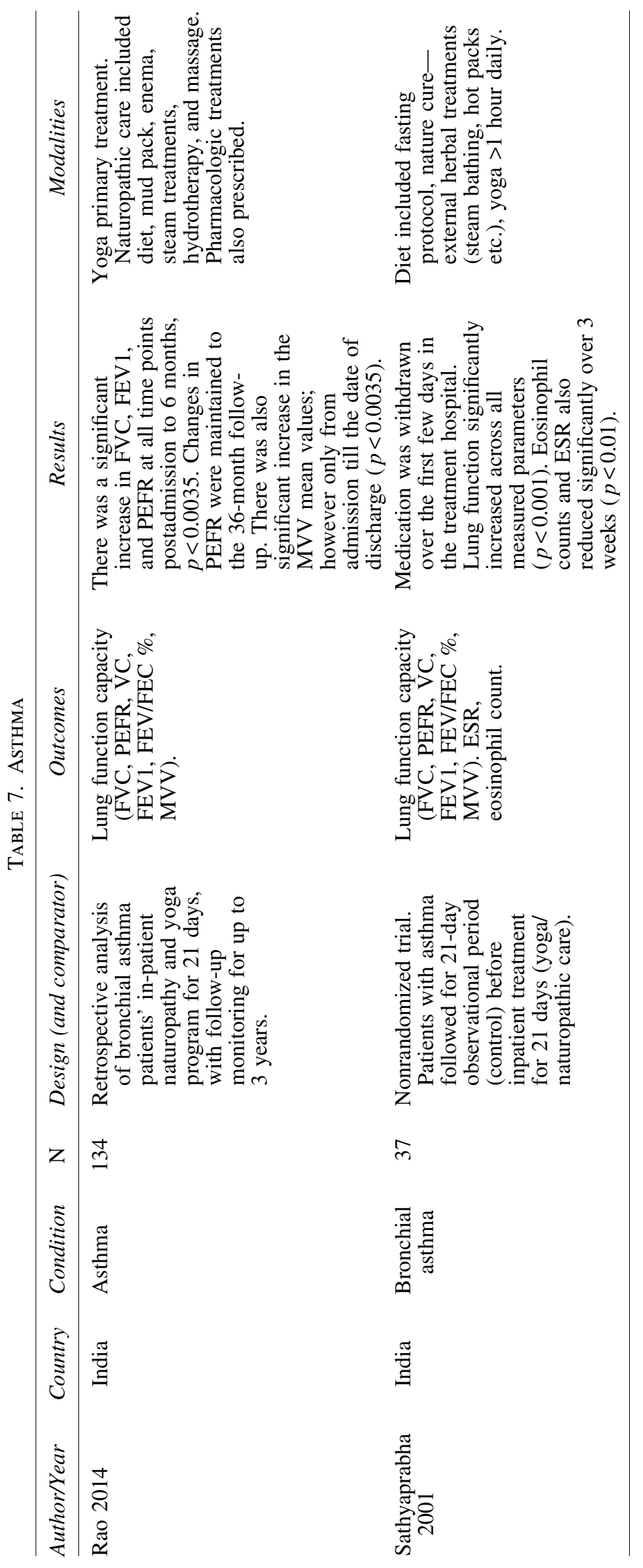




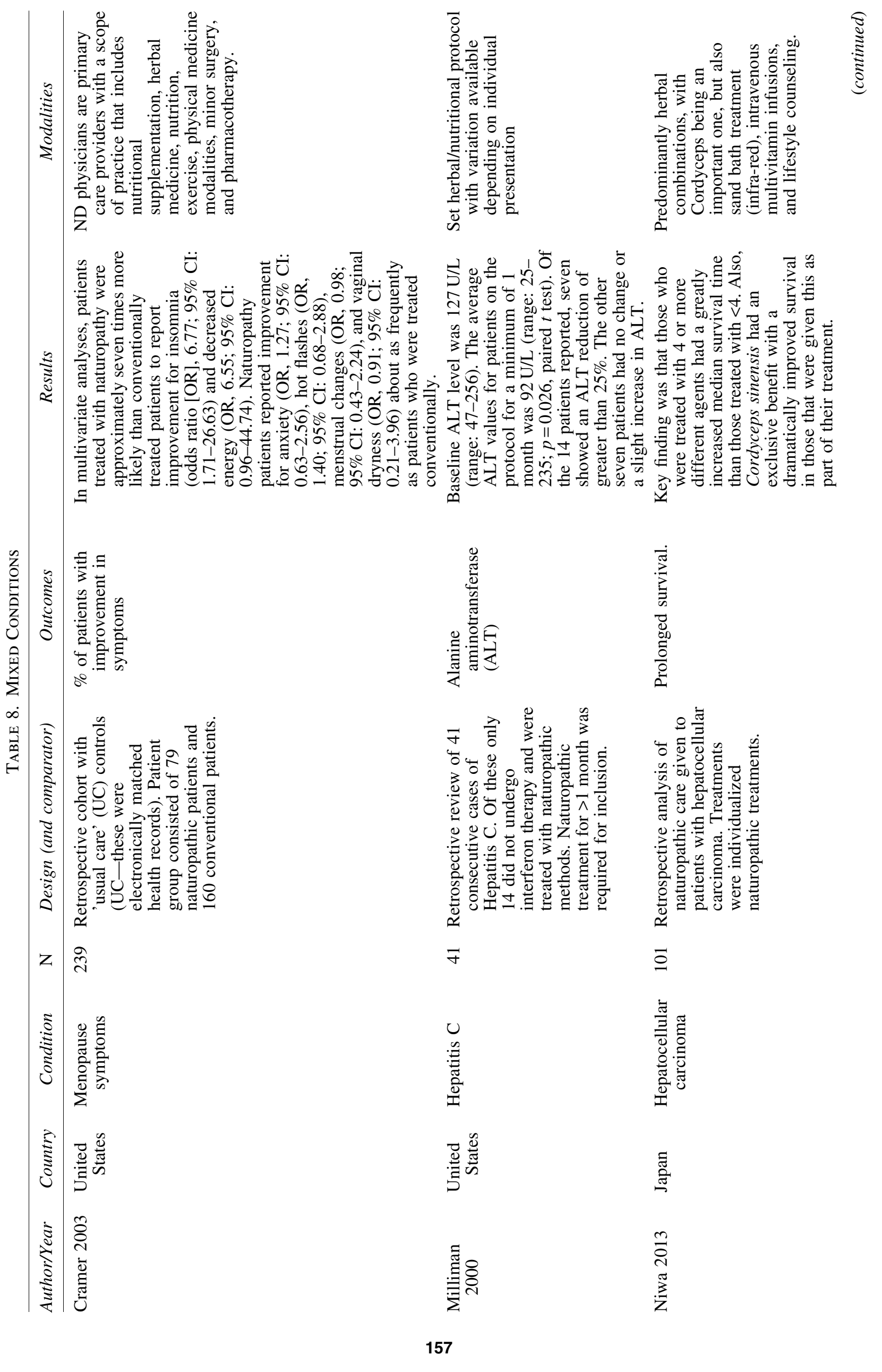




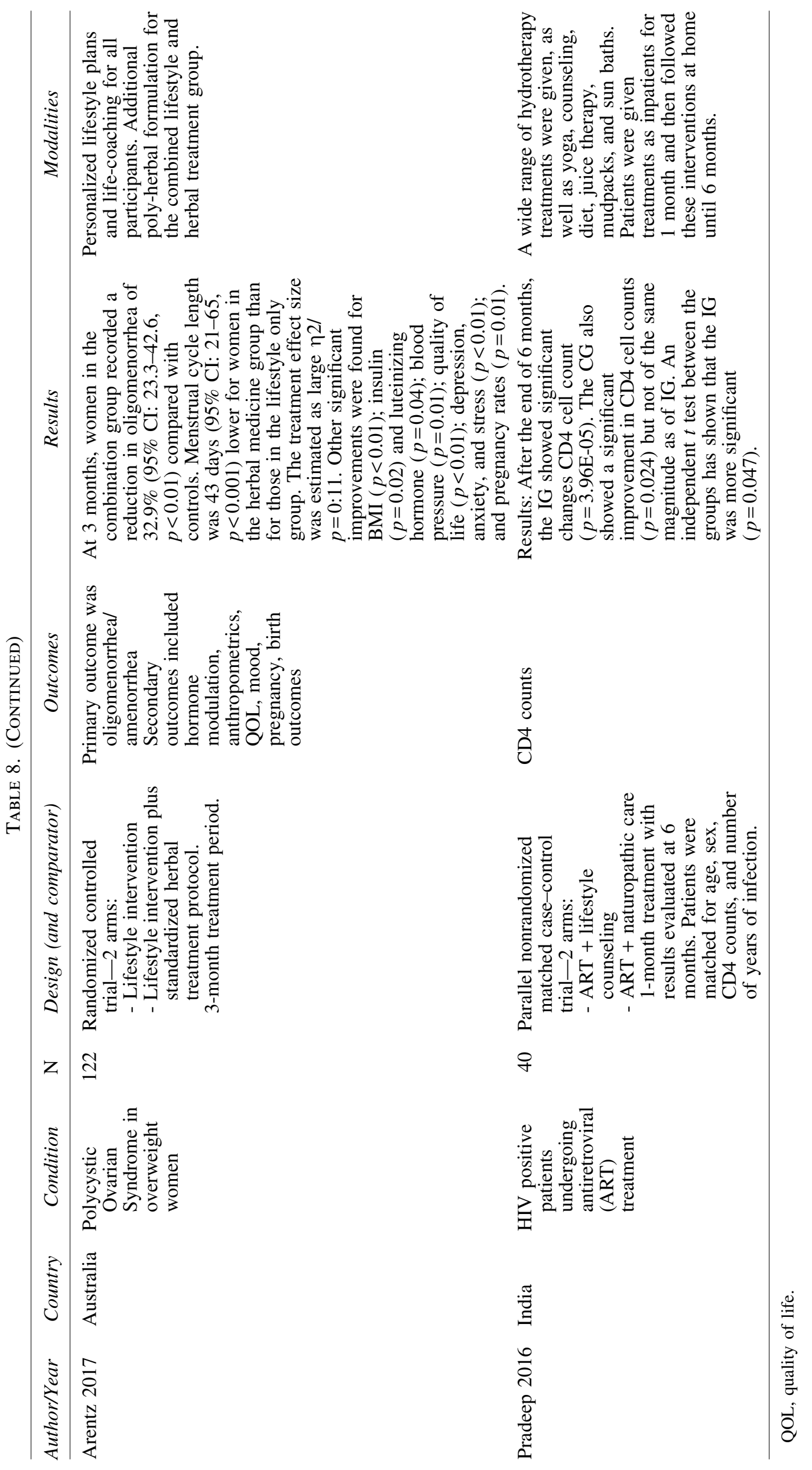




\begin{tabular}{|c|c|c|c|c|c|c|c|}
\hline & 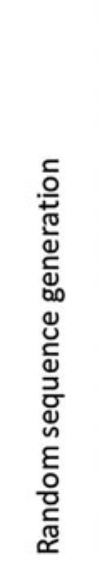 & 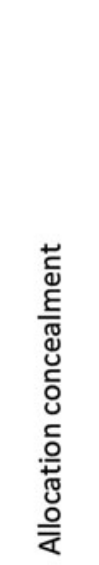 & 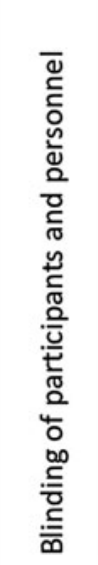 & 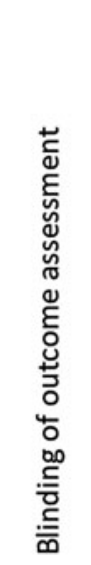 & 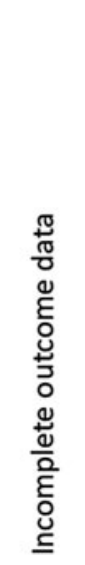 & 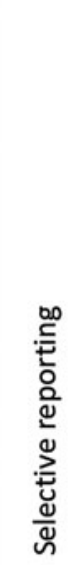 & 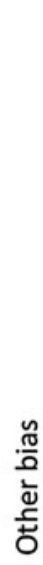 \\
\hline Seely (2013) & Low & High & High & High & Low & Low & $?$ \\
\hline Szczurko (2009) & Low & Low & High & Low & $?$ & Low & $?$ \\
\hline Shinto (2008) & High & High & High & $?$ & Low & $?$ & $?$ \\
\hline Ritenbaugh (2008) & Low & High & High & High & Low & Low & ? \\
\hline Szczurko (2007) & Low & High & High & Low & Low & Low & $?$ \\
\hline Cooley (2009) & Low & Low & High & Low & Low & Low & $?$ \\
\hline Tuet (2013) & Low & Low & High & High & Low & Low & ? \\
\hline Sathyaprabha (2001) & High & High & High & High & Low & Low & $?$ \\
\hline Arentz (2017) & Low & High & High & High & Low & Low & - \\
\hline
\end{tabular}

FIG. 3. Cochrane risk of bias assessment tables. gray, low risk of bias; light gray, unclear risk of bias; dark gray, high risk of bias

\begin{tabular}{|l|l|l|l|l|l|l|l|l|l|}
\hline Random sequence generation & & & & & & & & & \\
\hline Allocation concealment & & & & & & & & & \\
\hline Blinding of participants and personnel & & & & & & & & & \\
\hline Blinding of outcome assessment & & & & & & & & & \\
\hline Incomplete outcome data & & & & & & & & & \\
\hline Selective reporting & & & & & & & & & \\
\hline Other bias & & & & & & & & & \\
\hline
\end{tabular}

treatment in hospitals where naturopathic methods are used along with conventional medical methods. Four of the studies were observational, while two were RCTs. ${ }^{31,33}$ Naturopathic modalities included dietary interventions (including fasting), botanical medicine, physical therapy, and hydrotherapy as the main interventions, with additional homeopathy, ${ }^{31}$ acupuncture, ${ }^{14}$ and psychotherapy ${ }^{33}$ provided in one study each.

India. Seven studies from India ${ }^{13,36-41}$ included three in hypertension or CVD risk, ${ }^{13,37,38}$ two in asthma, ${ }^{39,40}$ one in type 2 diabetes, ${ }^{36}$ and one as an adjuvant to antiretroviral therapy. ${ }^{41}$ All of the Indian studies were inpatient treatments in either naturopathic hospitals or research institutes, with 15-30 days of care. These residential treatments could be classed as highly intensive, with yoga as the primary focus for all of the studies (being practiced up to four sessions per day). Naturopathic modalities included in the treatments varied, but consisted mainly of a specific vegetarian diet (often including a fasting protocol) and topical or in- halation applications that may or may not include botanical medicine. Pharmaceutic medication is also used as needed, with medication being concurrently reduced or withdrawn, as appropriate.

Australia. Three studies from Australia were identified. ${ }^{42-44}$ One of these was an observational pilot study of individualized naturopathic care for patients with anxiety and mood disorders conducted across private practice clinics $^{42}$ with most of the practitioners providing botanical and nutritional medicine along with diet and lifestyle advice. The second study was an open-label prospective cohort study of an integrative cardiac wellness program in patients undergoing cardiac surgery in a hospital setting, which was compared with a matched cohort of patients receiving "usual care", 43 and the third was a pragmatic clinical trial in private naturopathic practice. ${ }^{44}$ These latter two studies provided a set naturopathic protocol, including nutritional or botanical medicine in conjunction with personalized lifestyle and dietary planning. 
Table 9. Clinical Conditions and Study Design Index

\begin{tabular}{|c|c|c|c|c|c|c|c|}
\hline Condition & Year & Author & $R C T$ & $\begin{array}{c}\text { Noncontrolled } \\
\text { trial }\end{array}$ & $\begin{array}{l}\text { Prospective } \\
\text { cohort }\end{array}$ & $\begin{array}{l}\text { Retrospective } \\
\text { cohort }\end{array}$ & $\begin{array}{l}\text { Case } \\
\text { series }\end{array}$ \\
\hline \multirow[t]{7}{*}{ Musculoskeletal pain } & 2012 & Stange & & & & $\checkmark$ & \\
\hline & 2010 & Wiebelitz & & & $\checkmark$ & & \\
\hline & 2009 & Szczurko & $\checkmark$ & & & & \\
\hline & 2008 & Shinto & $\checkmark$ & & & & \\
\hline & 2008 & Ritenbaugh & $\checkmark$ & & & & \\
\hline & 2007 & Szczurko & $\checkmark$ & & & & \\
\hline & 2004 & Secor & & & $\checkmark$ & & \\
\hline \multirow[t]{6}{*}{ Cardiovascular disease } & 2016 & Edla & & & $\checkmark$ & & \\
\hline & 2014 & Braun & & & $\checkmark$ & & \\
\hline & 2013 & Seely & $\checkmark$ & & & & \\
\hline & 2012 & Nandakumar & & $\checkmark$ & & & \\
\hline & 2011 & Bradley & & & & $\checkmark$ & \\
\hline & 2011 & Murthy & & & $\checkmark$ & & \\
\hline \multirow[t]{4}{*}{ Diabetes } & 2016 & Bairy & & & $\checkmark$ & & \\
\hline & 2012 & Bradley & & & $\checkmark$ & & \\
\hline & 2009 & Bradley & & & & $\checkmark$ & \\
\hline & 2006 & Bradley & & & & $\checkmark$ & \\
\hline \multirow[t]{4}{*}{ Mood } & 2017 & Breed & & & $\checkmark$ & & \\
\hline & 2015 & Gurevich & & & & & $\checkmark$ \\
\hline & 2014 & Sarris & & & $\checkmark$ & & \\
\hline & 2009 & Cooley & $\checkmark$ & & & & \\
\hline \multirow[t]{2}{*}{ Asthma } & 2014 & Rao & & & & $\checkmark$ & \\
\hline & 2001 & Sathyaprabha & & $\checkmark$ & & & \\
\hline Polycystic ovarian syndrome & 2017 & Arentz & $\checkmark$ & & & & \\
\hline Cancer & 2013 & Niwa & & & & $\checkmark$ & \\
\hline Menopause & 2003 & Cramer & & & & $\checkmark$ & \\
\hline Hepatitis C & 2000 & Milliman & & & & & $\checkmark$ \\
\hline Multiple sclerosis & 2008 & Shinto & $\checkmark$ & & & & \\
\hline HIV & 2016 & Pradeep & & $\checkmark$ & & & \\
\hline \multirow[t]{5}{*}{ Mixed chronic conditions } & 2013 & Teut & $\checkmark$ & & & & \\
\hline & 2007 & Weidenhammer & & & $\checkmark$ & & \\
\hline & 2007 & Isbell & & & & $\checkmark$ & \\
\hline & 2002 & Ostermann & & & $\checkmark$ & & \\
\hline & 2001 & Beer & & & $\checkmark$ & & \\
\hline
\end{tabular}

RCT, randomized controlled trial.

United Kingdom. One study was identified from the United Kingdom, which was a retrospective evaluation of patient-assessed improvement in their primary symptom after treatment at a multidisciplinary complementary therapy clinic. ${ }^{45}$ The conditions were primarily musculoskeletal complaints and were treated with naturopathy, osteopathy, or CranioSacral therapy, with no distinction given between the different treatments in the report.

Japan. One study was identified from Japan, which was a retrospective analysis of all patients from a particular treatment center with hepatocellular carcinoma. Patients received individualized naturopathic treatment, including the use of medicinal mushrooms and other botanical medicines as the mainstay of treatment, with additional therapies, including intravenous multivitamin infusions and lifestyle counseling. ${ }^{46}$

\section{Clinical conditions, study design, settings, and modalities}

Included studies are categorized by clinical condition and study design (Table 9) and by clinical setting (outpatient and inpatient) and modalities (Table 10).
Cardiovascular disease. Six studies were assessed that investigated outcomes for present CVD or development of CVD risk factors, including two RCTs and four prospective cohort studies.

Four of these studies evaluated hypertension as a primary outcome.

- In India, results of two observational inpatient studies on populations with hypertension demonstrated substantive blood pressure control $(<140 / 90 \mathrm{mmHg})$ after naturopathic treatment. ${ }^{37,38}$ In one study $(n=104), 93 \%$ of participants achieved control after 21 days $(p<0.001),{ }^{38}$ while a separate study $(n=104)$ found that $99 \%$ of participants achieved control after 15 days $(p<0.001){ }^{37}$ In both studies, results were achieved in addition to simultaneous reduction or elimination of antihypertensive medication in a substantive number of the participants.

- A poster abstract from India reported a randomized waitlisted controlled clinical trial $(n=72),{ }^{13}$ which demonstrated an overall significant reduction $(p<0.05)$ in mean systolic blood pressure $(-15 \mathrm{mmHg} ; 140-$ $125 \mathrm{mmHg})$ and mean diastolic blood pressure $(-8 \mathrm{mmHg}$; $85-77 \mathrm{mmHg}$ ) after 3 weeks of intensive inpatient treatment. ${ }^{13}$ 


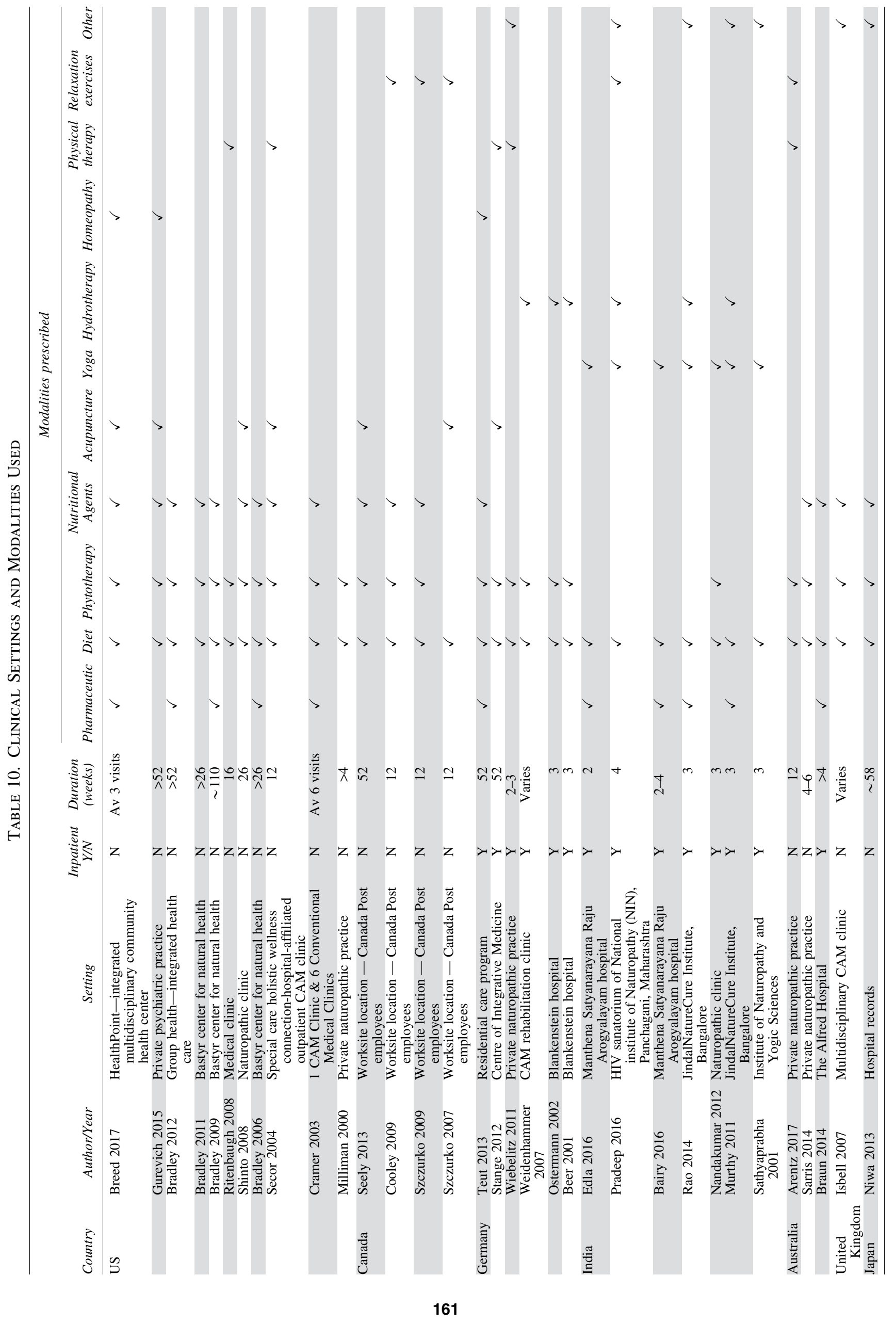


- A US retrospective analysis of outpatients $(n=85)$ treated for hypertension in a naturopathic clinic over 6 months showed a mean reduction of $26 \mathrm{mmHg}$ systolic and $11 \mathrm{mmHg}$ diastolic $(p<0.0001)$ in those with stage 2 hypertension and an overall increase from $14 \%$ at baseline to $44 \%$ of patients achieving blood pressure control $(<140 / 90 \mathrm{mmHg})$ over the 6-month treatment period. $^{23}$

Three studies examined multicomponent CVD risk as treatment outcomes. ${ }^{13,29,43}$

- An Australian prospective cohort study of patients undergoing cardiac surgery compared usual care $(n=585)$ against a standardized nutritional supplement treatment combined with individualized diet and lifestyle change $(n=337){ }^{43}$ Results showed significant postsurgery improvements in cardiac outcomes in the naturopathic care group and a $42 \%$ reduction in postsurgery inotropic support $(p<0.001)$.

- A Canadian RCT compared usual care against adjunctive naturopathic care for reducing CVD risk $(n=246){ }^{29}$ Results showed the 10-year Framingham CVD risk reduced by $-3.07 \%, p<0.001$ for the naturopathic over the "usual care" group after 52 weeks. This study also showed a lower frequency of metabolic syndrome $(-16.9 \%, p=0.002)$ in the naturopathic care cohort compared with usual care.

- In addition to the beneficial effects on blood pressure reported above, the randomized waitlisted controlled clinical trial $(n=72)^{13}$ from India showed reductions in blood glucose, LDL cholesterol, and triglycerides after 3 weeks of residential naturopathic treatment.

Overall, these studies show naturopathic treatment results in a clinically significant benefit for treatment of hypertension, reduction in metabolic syndrome parameters, and improved cardiac outcomes postsurgery.

Type 2 diabetes. Four studies on type 2 diabetes mellitus were assessed, including two retrospective and two prospective cohort studies.

In the US, two retrospective studies and one prospective cohort study by the same research group from Bastyr University $^{20-22}$ focused on blood glucose management.

- In the initial retrospective study, all subjects $(n=16)$ received 6 months or more of naturopathic care. Results showed that $31 \%$ of patients achieved blood glucose control (HbA1c <7\%) and $61 \%$ achieved moderate control (HbA1c 7\%-10\%). ${ }^{21}$

- A second retrospective study $(n=37)$ showed reduced HbA1c of $-0.65 \%(p=0.046)$, with a mean duration of care of 27 months. ${ }^{20}$ Other significant positive changes were demonstrated for blood pressure $(-7 \mathrm{mmHg}$ systolic, $p=0.02$; and $-5 \mathrm{mmHg}$ diastolic, $p=0.003$ ) and triglycerides $(-45 \mathrm{mg} / \mathrm{dL}, p=0.037)$, with no difference in cholesterol. ${ }^{20}$

- A prospective study $(n=40)$ showed reduction in HbA1c of $-0.90 \%(p=0.02)$ at 6 months after the initial visit. $^{22}$

- One prospective cohort study in India $(n=101)^{36}$ examined inpatient naturopathic treatment for 15-30 days, with patients reviewed again at 3 months. Findings included significant mean reductions of $-0.9 \%$ in HbA1c $(p<0.001)$ after the initial treatment period and a reduction of $-1.7 \%$ at 3 months for those with excellent adherence to the treatment provided.

Overall, these studies show naturopathic treatment results in a significant benefit for treatment of diabetes, with reductions in $\mathrm{HbA} 1 \mathrm{c}$ that are clinically relevant.

Musculoskeletal pain. Six studies of musculoskeletal pain (five articles and one abstract) were included. Clinical conditions included chronic back pain, rotator cuff tendinitis, multiple sclerosis, temporomandibular disorder (TMD), and generalized chronic body pain. Two studies were conducted in Germany, ${ }^{14,33}$ three in the US, ${ }^{17-19}$ and two in Canada. $^{26,27}$

- A Canadian RCT compared 12 weeks of naturopathic care with standard physical therapy $(n=75)$ in employees of Canada Post who had chronic lower back pain. Participants in the naturopathic care cohort showed significant improvement $(p<0.0001)$ in back pain compared with patients receiving standard physical therapy. ${ }^{26}$

- A German controlled prospective cohort study compared naturopathic care with orthopedic care $(n=348)$ in adults who had chronic back pain requiring inpatient treatment. This study showed no differences between naturopathic and standard orthopedic treatment in the whole study population. However, at 3 months, a significant improvement $(p=<0.014)$ was found in a subgroup of women receiving naturopathic medicine ( $86 \%$ of the naturopathic patients), compared with orthopedic care. $^{33}$

- Also in Germany, a poster abstract reported a prospective clinical trial in chronic musculoskeletal pain (including back pain), conducted over 2 weeks of inpatient treatment $(n=221)$. Naturopathic care decreased mean pain scores by 15.1 from $60.7 \pm 23.0$ (admission) to $45.6 \pm 26.2$ (1 year post-treatment) $(p<0.0001) .^{14}$

- In Canada, a RCT conducted in rotator cuff tendinitis $(n=85)$ compared a set naturopathic treatment protocol to a standardized exercise routine/placebo supplementation over 12 weeks. Naturopathic treatment decreased the Shoulder Pain and Disability Index by $54.5 \%$ $(p<0.0001)$ compared to $18 \%(p=0.0241)$ in the control group. ${ }^{27}$

- In the US, a multiple sclerosis RCT $(n=45)$ compared naturopathic treatment with "usual care" and with "usual care plus education". They found no significant differences between groups on any outcome measure at 6 months. ${ }^{19}$

- Another US RCT investigated naturopathic treatment in comparison to Traditional Chinese Medicine (TCM) and specialized dental care $(n=160)$ for TMD. For worst pain, the improvement in the naturopathic medicine group was statistically significant compared to specialized dental care $(-1.02 \pm 0.45, p=0.025)$. Naturopathic treatment provided significantly greater decreases than either TCM $(p<0.034)$ or specialized dental care $(p<0.012)$ in TMD-related psychosocial interference. ${ }^{17}$

- Also in the US, subjects with pain from any cause as a feature of presentation were randomized to acupuncture, chiropractic, or naturopathic medicine treatment 
$(n=94)$. Naturopathic treatment significantly reduced pain from baseline to end of treatment $(p<0.0001)$, but was not statistically significantly different from the other groups. ${ }^{18}$

Overall, this diverse group of studies shows that naturopathic treatment decreased pain scores to a degree comparable or better than standard care or other active treatment controls.

Mood disorders. Four studies reported on naturopathic treatment for mood disorders: one in anxiety, one in depression, one on both anxiety and depression, and another on bipolar disorder.

- A Canadian RCT randomized patients to standardized psychotherapy with or without naturopathic care for moderate-to-severe anxiety $(n=75){ }^{28}$ Beck Anxiety Inventory scores decreased by $56.5 \%(p<0.0001)$ in the naturopathic treatment group compared to $30.5 \%$ $(p<0.0001)$ in the psychotherapy-only group.

- A US prospective cohort study $(n=60)$ showed significant improvements using naturopathic treatment, with symptomatic improvement in depression (16.4 vs. $8.6, p<0.0001)$ and anxiety $(12.4$ vs. $7.2, p<0.0001)$ scores in patients who returned for two or more visits. ${ }^{15}$

- A small Australian observational study ${ }^{42}(n=8)$ showed improved scores on all areas of the Depression Anxiety Stress Scale-21 (DASS-21) and significant improvement in clinical outcomes with naturopathic treatment in patients who returned for two or more visits $(p<0.005)$.

- In the US, a retrospective case series reported on a small subset of patients diagnosed with treatment-resistant bipolar disorder $(n=7)$ receiving $>1$ year of naturopathic treatment. ${ }^{16}$ Results were varied, but showed mood stabilization along with withdrawal of psychotropic medication.

Overall, significant reductions in anxiety and depression levels were shown across this group of studies.

Complex chronic disease. A total of five studies were included for complex chronic disease, comprising a broad group of mixed chronic conditions within individuals. The primary outcomes included changes in QOL and symptom scores.

- In Germany, three prospective cohort studies examined effectiveness of naturopathic treatment outcomes over a broad group of mixed chronic conditions, in terms of QOL outcomes. ${ }^{32,34,35}$ The studies all used different outcome measures to evaluate treatment effectiveness, with all showing positive results for improving QOL. In one study, the intensity of the main complaint decreased from $59 \pm 25$ by an average of 25 points at discharge (reduced to 19 points at 6-month follow-up) corresponding to an effect size of 0.86 (0.62 at 6-month follow-up). ${ }^{32}$ The other two studies report statistically significant increases across the majority of QOL domains as measured by two independent QOL scales $(p<0.01)^{34,35}$

- Also in Germany, a cluster-randomized trial $(n=58)$ of naturopathic care compared with usual care in aged residential community living $^{31}$ showed a small-tomedium effect size on a range of geriatric QOL scales.

- In the United Kingdom (UK), a retrospective evaluation of clinic outpatients $(n=49)$ receiving naturopathic care over a 3 -year period ${ }^{45}$ showed improvement $(p<0.001)$ between the first and last consultation in patients' symptom scores.

While difficult to group together, this varied group of studies shows an overall positive effect on QOL and symptomatic improvement with naturopathic care.

Asthma. Two studies of naturopathic treatment for asthma were included. ${ }^{39,40}$ Both studies were conducted in India and both showed that naturopathic inpatient treatment improved clinical asthma profiles.

- A nonrandomized crossover trial $(n=37)^{40}$ examined 21 days of standard drug therapy at home followed by, and compared to, 21 days of intensive naturopathic inpatient treatment. Results showed significant increase across all measured lung function parameters $(p<0.001)$ and reduced eosinophil counts after naturopathic treatment $(p<0.01) .^{40}$

- A retrospective evaluation of patients $(n=134)^{39}$ also investigated intensive naturopathic inpatient treatment for 21 days and showed a significant increase in some indices of lung function at all time points, from postadmission to 6 months $(p<0.0035),{ }^{39}$ with increased peak expiratory flow rate maintained to a 36-month follow-up.

Overall, these two Indian studies show significant positive results for intensive inpatient naturopathic treatment of asthma in lung function parameters.

Mixed conditions. This group contains conditions where only a single study was found. The areas of these conditions are as follows: cancer ${ }^{46}$ menopause, ${ }^{25}$ hepatitis $\mathrm{C},{ }^{24}$ multiple sclerosis, ${ }^{19}$ polycystic ovary syndrome (PCOS) ${ }^{44}$ and HIV (as an adjuvant to antiretroviral therapy). ${ }^{41}$

- In liver cancer, a single retrospective analysis of naturopathic treatment in Japan $(n=101)$ showed a dramatically improved survival rate when Cordyceps sinensis was a component of the multi-modality treatment administered. ${ }^{46}$

- In a retrospective cohort study on menopause in the US $(n=79)$, researchers compared naturopathic treatment with usual care $(n=160)$. The most significant improvements resulting from naturopathic treatment occurred for insomnia and decreased energy, with a sevenfold improvement over usual care. Improvements in other symptoms were comparable with improvements in the control group. ${ }^{25}$

- In a retrospective case series of patients with Hepatitis $\mathrm{C}$ undertaking naturopathic treatment $(n=14)$, all participants showed reductions in serum alanine aminotransferase (ALT) (average $35 \mathrm{U} / \mathrm{L}$ ). Seven cases showed an ALT reduction of more than $25 \% .^{24}$

- An RCT in multiple sclerosis found no significant differences between usual care, usual care plus education, and usual care plus naturopathic treatment. ${ }^{19}$ However, statistical trends favoring the naturopathic 
treatment group were found in a single subscale of SF36 (general health) and in the timed-walk and symptoms of neurologic impairment (EDSS).

- An RCT in overweight PCOS patients $(n=122)$ found highly significant improvement $(p<0.001)$ in the primary outcome of oligomenorrhea/amenorrhea after 3 months of treatment. ${ }^{44}$ The trial compared effect of a LI alone, with LI plus a botanical medicine protocol. Menstrual cycle length was 43 days lower for women in the herbal medicine group (95\% CI: $21-65, p<0.001)$ than for those in the lifestyle-only group, with a large effect size. Improvements across other areas, including BMI, insulin, hormone levels, stress, and pregnancy, also were seen.

- A prospective, parallel, matched-control study of naturopathic and yoga interventions as adjuvant treatments to antiretroviral therapy in a group with HIV, conducted over 1 month, showed improved CD4 count in the intervention group over the control group which received only antiretroviral therapy $(p=0.047){ }^{41}$

Heterogeneity. Clinical heterogeneity (defined as differences in participants, treatments, outcome characteristics, or research setting) ${ }^{47}$ in this scoping review is substantial. While all the interventions are similar in intervention type (whole-system, multi-modality naturopathic medicine), they vary substantively at the patient level (condition, baseline severity, age, gender, ethnicity, and comorbidities); intervention level (duration and comparator/controls); outcome level (outcome measure and definitions); and in research setting. The authors did not test for statistical heterogeneity.

Study quality and risk of bias. There is a wide range of quality in the included studies, given the breadth of the research reported. Cochrane risk of bias assessments were completed on the nine RCTs (Fig. 3), showing low risk of bias for all areas, except blinding of participants and personnel, and moderate bias for allocation concealment. In observational research, selection bias is considered high for several studies in which retrospective data have been reported and where outcomes include only patients who have returned for multiple visits. Reporting bias also is likely to be high in retrospective studies in which isolated outcomes are reported. Several of the prospective cohort studies are well-conducted, with a low level of bias, particularly those, such as Braun et al., ${ }^{43}$ Teut et al., ${ }^{31}$ and Bradley et al., ${ }^{22}$ that compared results with a "usual care" cohort.

\section{Discussion}

This systematic scoping review identified a diverse collection of quantitative whole-system, multi-modality naturopathic medicine research from around the world reported in 33 publications. A majority of the research (15 articles) was conducted in North America, where the modern naturopathic medical profession has developed ${ }^{48}$ and where the first school of naturopathic medicine was founded by Benedict Lust in 1902. ${ }^{1}$ Other countries contributing research include Germany (six studies), where naturopathy is rooted in the development of hydrotherapy by founders Priessnitz and Kneipp, ${ }^{49}$ and India (six articles) where naturopathy was popularized and influenced by MK Gandhi, regarded as the Father of the Indian nation, at the turn of the 20 th century. ${ }^{50}$

\section{Clinical outcomes}

Although results from these studies are highly diverse, they also are predominantly positive, showing improved health outcomes and QOL across conditions and across nationalities. These studies demonstrate a broad range of naturopathic modalities, against a background of different practitioner training, legislative and regulatory jurisdictions, and different research approaches. Their results concur with Oberg et al. ${ }^{9}$ who determined the effect sizes of the primary medical outcomes for 13 North American studies, concluding that there were positive outcomes and improved QOL in individuals with, or at risk for, chronic conditions, including CVD, ${ }^{23,29}$ type 2 diabetes, ${ }^{20-22}$ chronic pain, ${ }^{17,18,26,27}$ anxiety, ${ }^{28}$ hepatitis $\mathrm{C},{ }^{24}$ and menopausal symptoms. ${ }^{25}$ The authors have updated and expanded this review to the global literature, thereby increasing the range of positive outcomes to include depression and anxiety, ${ }^{15}$ bipolar disorder, ${ }^{16}$ asthma, ${ }^{39,40}$ PCOS, ${ }^{44}$ and increased cancer survival time. ${ }^{46}$ It also adds additional studies to support positive outcomes for CVD, ${ }^{13,37,38,43}$ type 2 diabetes, ${ }^{36}$ chronic pain, ${ }^{14,32}$ and anxiety and mood disorders. ${ }^{42}$

Three of the German studies examined mixed chronic conditions and demonstrated positive outcomes for QOL and perceived pain in: older adults (mean age 79.4 years) living in nursing homes ${ }^{31}$ and older adults (mean age 57.3 years) admitted to hospital for allergic complaints and rheumatic, chronic-bronchial, and metabolic diseases. ${ }^{34,35}$ In addition, one UK study of mixed chronic conditions demonstrated positive outcome for overall symptom improvement. ${ }^{45}$

The benefit of naturopathic treatment as an adjunct to antiretroviral treatment in individuals with HIV could not be assessed, because the study ${ }^{41}$ lacked the data required to make a clinical assessment of the effect.

The study on multiple sclerosis ${ }^{19}$ showed no difference in the primary outcome (the QOL short form 36; SF-36) between the three intervention groups (usual care, usual care plus naturopathic care, or usual care plus education). Shinto et al. ${ }^{19}$ concluded that positive outcome trends in individuals with multiple sclerosis warranted further evaluation.

\section{Community versus inpatient studies}

A main characteristic of the North American research is that all the studies were undertaken in free-living individuals treated in a community setting. By comparison, all six of the Indian studies, a single UK study, and five of the six German studies were undertaken in inpatients admitted to a treatment facility. The three Australian studies were mixed, with two in community practice and one in an inpatient setting (Table 9).

In Germany, naturopaths (heilpraktikers) are licensed by the state and comprise 40,000 of the 60,000 naturopathic professionals in Europe. ${ }^{51}$ Heilpraktikers are nonmedical practitioners trained in the philosophy and modalities of naturopathic medicine and trace their roots to the origin of the profession. To date, no research into whole-system naturopathic medicine by Heilpraktikers could be located with an English title and abstract. In addition to Heilpraktikers, there is a group of medical doctors (naturheilkunde) who specialize in naturopathic modalities and self-identify as providing naturopathic treatment. ${ }^{49}$ All studies from Germany included in this review were undertaken by these 
naturopathic practitioners in both inpatient and in residential facilities.

A meta-analysis ${ }^{52}$ of eight studies on the effect of German inpatient integrative medicine research on QOL included four of the studies included in this scoping review. A random effect meta-analysis of the eight studies revealed an overall effect size of 0.37 (95\% CI: $0.28-0.45)$ in the physical score and 0.38 (95\% CI: $0.30-0.45)$ in the mental score of the SF-36, demonstrating the effectiveness of the inpatient treatment model used in Germany.

\section{Different countries, different designs, similar conclusions}

An insight into the diversity of naturopathic treatment, and the outcomes that can be achieved, can be gained by contrasting two different studies on chronic lower back pain. One study was undertaken in $\mathrm{Canada}^{26}$ in an outpatient setting in individuals with chronic lower back and the other in Germany ${ }^{33}$ in a cohort who required hospitalization for chronic lower back pain.

The Canadian naturopathic treatment consisted of acupuncture, relaxation techniques, and dietary recommendations (diet high in omega three fatty acids, magnesium, and calcium). In Germany, classical naturopathic treatment is codified for use in acute inpatient settings with a minimum requirement that five of these eight therapies are applied: (1) nutrition therapy; (2) hydrotherapy/thermotherapy; (3) other physical modalities; (4) phytotherapy; (5) lifestyle-regulatory therapy; (6) exercise therapy; (7) detoxification procedures; or (8) an additional procedure (manual therapy, acupuncture/ Chinese medicine, homeopathy, neural therapy, or art/music therapy). ${ }^{53}$

Both studies used the Oswestry Disability Index as the primary outcome measure and demonstrated that wholesystem multi-modality naturopathic medicine made a significant difference in comparison to controls. The Canadian study demonstrated that naturopathic treatment is more effective than education and exercise for chronic lower back pain. The German study demonstrated that it is comparable with mainstream orthopedic treatment when back pain is so acute as to warrant inpatient care and that it is potentially better than mainstream orthopedic care for women. Together, these two very different studies demonstrate a stronger case for the effectiveness of whole-system multi-modality naturopathic treatment of chronic lower pain.

\section{Regional differences and generalizability}

The WNF has determined that there is a high degree of global consistency in the core concepts that define naturopathic medicine and that all countries utilize a common set of naturopathic modalities. ${ }^{54,55}$ The consistent positive outcomes in similar conditions in different countries are likely to reflect this commonality.

Regional differences, however, may affect the generalizability of studies, if the scope of practice used in a specific study includes treatment modalities that are not accessible or utilized in other regional areas for historical, legal, or educational reasons.

Specific regional differences exist in naturopathic practice concerning modalities emphasized (e.g., the addition of acupuncture in Canada or pharmaceutic prescribing rights in some areas of the US). In some jurisdictions, there are core modalities underlying naturopathic practice, such as yoga in India or the combination of osteopathic techniques with naturopathic practice in the UK. Thus, the UK study on the treatment of musculoskeletal conditions using a mixture of osteopathic and naturopathic techniques ${ }^{45}$ is consistent with naturopathic practice in that country.

The diverse practice settings and the extensive range of modalities represented in the research provide a sound argument for expanding the scope of practice in jurisdictions where generalizability is limited. The extent to which the studies included in this scoping review are generalizable to other countries must be assessed on a study-by-study basis.

\section{Pragmatic trials versus therapeutic tools}

Pragmatic whole-system (whole-practice) research provides a "real life" snapshot of how naturopathic medicine is practiced in the community, reflecting the naturopathic individualized approach to treatment and ongoing management. In the absence of whole-system data, the only way to effectively and objectively evaluate a discipline is to assess its major therapeutic tools. In naturopathic medicine this would include evidence for dietary and lifestyle interventions and specific botanical medicines and nutritional supplements.

In 2005, a review was undertaken of naturopathy and of Western herbal medicine in Australia. ${ }^{5}$ The report concluded that while evidence for the whole-system practice of naturopathic medicine was lacking, a range of nutritional supplements and botanical medicines (the "tools of trade") demonstrated benefits at the highest levels of evidence and have proven efficacy.

In conventional medicine, evidence for the effectiveness of their "tools of trade" (pharmaceutics and surgery) is generally considered sufficient to demonstrate the effectiveness of its practice. By comparison, a recent governmental review of naturopathic medicine in Australia judged the practice of naturopathic medicine solely on the scope of whole-system research, limited to only systematic reviews containing RCTs published since 2008. ${ }^{11}$ Based on this limited scope, they concluded that naturopathic medicine's overall effectiveness could not be proven, and the Government has proposed exclusion from private health insurance effective from April 1st, 2019.

To provide a more comprehensive method for assessing the effectiveness of naturopathic medicine, there is a real need for a new type of effectiveness review. Such a review would systematically evaluate evidence for specific therapeutic agents used by naturopathic clinicians combined with the results of pragmatic clinical trials on whole-system naturopathic practice, in a specific condition or population. It is not sufficient, nor appropriate, to rely on either aspect alone as the sole method of assessment of the effectiveness of naturopathic medicine.

\section{Context of EBM}

The EBM movement began in conventional medicine due to a concern that clinical decision-making was not evidence based. ${ }^{56}$ The scope of conventional medicine is so large that charting the extent of its total evidence at any given point in time is problematic. In 2007, BMJ Clinical Evidence reviewed 2500 treatments supported by good evidence. It 
rated $15 \%$ of treatments as beneficial, $22 \%$ as likely to be beneficial, $7 \%$ as partly beneficial and partly harmful, 5\% unlikely to be beneficial, and $4 \%$ likely to be ineffective or harmful. For the remaining $47 \%$, the effect of treatment was rated as currently not demonstrated. ${ }^{57}$ If this review was undertaken today, these numbers would differ; however, it is important to recognize from these figures that conventional medicine, like all fields in health care, has extensive work to do regarding the evidence on which practice is based.

In contrast to classical RCTs which have dominated EBM and which utilize a reductionist approach that fails to recognize the complexities of real-world clinical practice, ${ }^{2}$ the research outlined in this scoping review is pragmatic in nature and sets out to determine the effectiveness of a whole-system approach in real-world clinical practice.

\section{Limitations}

There are several limitations inherent in grouping such as a broad range of heterogeneous studies. No specific analysis of the effectiveness of naturopathic treatment was conducted, due to the breadth of study types, outcomes assessed, treatment settings, and modalities used. The most robust studies reported results in comparison with usual care; however, comparative controls were used in only 4 of 12 prospective studies ${ }^{18,22,33,43}$ and in one of eight retrospective studies. ${ }^{25}$ In addition, there is a high risk of reporting and selection bias in many of the observational studies (i.e., criteria such as multiple return visits to naturopathic centers for patient inclusion in the dataset).

The types of modalities used and the intensity of treatments are highly variable across the studies reported here. It is not possible to compare outcomes for intensive inpatient treatment with several visits spaced over 6 months in a community setting. As such, the aim of this scoping report was not to compare the effectiveness of the research, but to show the breadth of the research into whole-system naturopathic medicine.

There is little distinction between some included studies where "integrative medicine" is applied and some of the excluded studies using integrative medicine. The acceptance criterion was that treatments were administered by a selfidentified naturopath, as opposed to a conventional doctor or nurse; however, the authors recognize that some studies of integrated hospital care might not represent naturopathic medicine as clearly as studies that have naturopathic-only treatment. Also some studies of treatment provided by naturopaths might have been overlooked if this was not specified in the text. In addition, inclusion criteria for non-English language articles might have precluded relevant studies.

The decision to limit inclusion of case series to those with five or more cases was arbitrary and might have excluded some studies. Although seven was the median number of cases included in articles with titles specifying "case series," the number of cases included is not a differentiating characteristic. ${ }^{58}$ The decision was taken to ensure that any inference about clinical practice was based on multiple observations.

The majority of studies assessed were positive, which carries a specific concern regarding publication bias (the possibility that negative studies might have been undertaken, but not reported). This is known to be true in pharmaceutic research, ${ }^{59}$ but currently difficult to assess in naturopathic medicine.

\section{Conclusions and Future Directions}

The global naturopathic research landscape contains a small, but expanding body of practice-based, whole-system, multi-modality research. To date, research with higher methodological quality shows that whole-system multi-modality naturopathic medicine is effective for treating a range of conditions, including cardiovascular disorders, musculoskeletal pain, type 2 diabetes, PCOS, depression, and anxiety. Research with lower methodological quality also suggests that naturopathic medicine is effective for treating chronic pain, hepatitis $\mathrm{C}$, menopausal symptoms, bipolar disorder, and asthma and in increasing cancer survival time. Results were positive across world regions for similar conditions, which are likely to reflect the global consistency in applying the core concepts of naturopathic practice utilizing the common set of naturopathic modalities.

Although there is a vast array of clinical trial evidence supporting the tools of trade used in naturopathic medicine (dietary and lifestyle interventions and specific botanical medicines and nutritional supplements), there is a distinct lack of well-conducted pragmatic trials evaluating the complex intervention of whole-system, multi-modality naturopathic care. Until substantively more whole-system research is undertaken, evaluating the effectiveness of naturopathic medicine requires a combination of both these types of evidence.

There is a need for pragmatic, real-world trials in which complex naturopathic treatment is compared with usual care to build a high-quality evidence base on the effectiveness of whole-system, multi-modality naturopathic practice. This need has recently led to development of a research consortium of naturopathic academic clinics in four countries and across multiple world regions to develop robust, international, multicenter collaboration. ${ }^{60}$ This consortium has been endorsed by the World Naturopathic Federation, with the goal to significantly increase the amount and quality of global naturopathic whole-system research.

\section{Author Disclosure Statement}

No competing financial interests exist.

\section{References}

1. Myers SP, Hunter A, Snider P, Zeff J. Naturopathic Medicine. In: Robson $\mathrm{T}$, ed. Introduction to Complementary Medicine. Sydney: Allan and Unwin, 2003.

2. Wardle J, Oberg EB. The intersecting paradigms of naturopathic medicine and public health: Opportunities for naturopathic medicine. J Altern Complement Med 2011;17: 1079-1084.

3. World Health Organisation. WHO Traditional Medicine Strategy 2014-2023. World Health Organization, Geneva, Switzerland 2013.

4. Margolis SA, Russell B. Evidence based medicine. Evidence-Based Medicine Editorial 2018;47:6.

5. Lin V, Bensoussan A, Myers S, et al. The Practice and Regulatory Requirements of Naturopathy and Western Herbal Medicine. Melbourne: School of Public Health, Latrobe University; 2005.

6. Jagtenberg T, Evans S, Grant A, et al. Evidence-based medicine and naturopathy. J Altern Complement Med 2006;12:323-328.

7. Ooi SL, Rae J, Pak SC. Implementation of evidence-based practice: A naturopath perspective. Complement Ther Clin Pract 2016;22:24-28. 
8. Standish LJ, Calabrese C, Snider P. The naturopathic medical research agenda: The future and foundation of naturopathic medical science. J Altern Complement Med 2006;12:341-345.

9. Oberg E, Bradley R, Cooley K, et al. Estimated effects of whole-system naturopathic medicine in select chronic disease conditions: A systematic review. Altern Integr Med 2015;4:192.

10. Levac D, Colquhoun H, O'Brien KK. Scoping studies: Advancing the methodology. Implement Sci 2010;5:69.

11. Baggoley C. Review of the Australian Government Rebate on Natural Therapies for Private Health Insurance. Australia Government Department of Health, Australia, 2017.

12. Higgins JPT, Altman DG, Gøtzsche PC, et al. The Cochrane Collaboration's tool for assessing risk of bias in randomised trials. BMJ 2011;343:d5928.

13. Nandakumar B, Kadam A, Srikanth H, Rao R. P02.50. Naturopathy and yoga based life style intervention for cardiovascular risk reduction in patients with cardiovascular risk factors: A pilot study. BMC Complement Altern Med 2012;12(Suppl 1):1-1.

14. Stange R, Hackermeier U, Franzen G, et al. P02.172. 1-year sustaining efficacy of multidimensional therapy for inpatients with different conditions of chronic musculoskeletal pain. BMC Complement Altern Med 2012;12(Suppl 1): $\mathrm{P} 228-\mathrm{P} 228$.

15. Breed C, Bereznay C. Treatment of Depression and Anxiety by Naturopathic Physicians: An Observational Study of Naturopathic Medicine Within an Integrated Multidisciplinary Community Health Center. J Altern Complement Med 2017; 23:348-354.

16. Gurevich MI, Robinson CL. Medication-free Alternatives for Long-term Maintenance of Bipolar Disorder: A Case Series. Glob Adv Health Med 2015;4:53-60.

17. Ritenbaugh C, Hammerschlag R, Calabrese C, et al. A pilot whole systems clinical trial of traditional Chinese medicine and naturopathic medicine for the treatment of temporomandibular disorders. J Altern Complement Med 2008;14:475-487.

18. Secor ER, Markow MJ, Mackenzie J, Thrall RS. Implementation of outcome measures in a complementary and alternative medicine clinic: Evidence of decreased pain and improved quality of life. J Altern Complement Med (New York, N.Y.) 2004;10:506-513.

19. Shinto L, Calabrese C, Morris C, et al. A randomized pilot study of naturopathic medicine in multiple sclerosis. J Altern Complement Med 2008;14:489-496.

20. Bradley R, Kozura E, Buckle H, et al. Description of clinical risk factor changes during naturopathic care for type 2 diabetes. J Altern Complement Med (New York, N.Y.) 2009;15:633-638.

21. Bradley R, Oberg EB. Naturopathic Medicine and Type 2 Diabetes: A Retrospective Analysis from an Academic Clinic. Altern Med Rev 2006;11:30.

22. Bradley R, Sherman KJ, Catz S, et al. Adjunctive naturopathic care for type 2 diabetes: Patient-reported and clinical outcomes after one year. BMC Complement Altern Med 2012;12:44-44.

23. Bradley R, Kozura E, Kaltunas J, et al. Observed Changes in Risk during Naturopathic Treatment of Hypertension. Evid Based Complement Altern Med 2011;2011:826751826751.

24. Milliman WB, Lamson DW, Brignall MS. Hepatitis C; a retrospective study, literature review, and naturopathic protocol. Altern Med Rev 2000;5:355-371.
25. Cramer EH, Jones $\mathrm{P}$, Keenan NL, Thompson BL. Is naturopathy as effective as conventional therapy for treatment of menopausal symptoms? J Altern Complement Med (New York, N.Y.) 2003;9:529-538.

26. Szczurko O, Cooley K, Busse JW, et al. Naturopathic care for chronic low back pain: A randomized trial. PLoS One 2007;2:e919-e919.

27. Szczurko O, Cooley K, Mills EJ, et al. Naturopathic treatment of rotator cuff tendinitis among Canadian postal workers: A randomized controlled trial. Arthritis Rheum 2009;61:1037-1045.

28. Cooley K, Szczurko O, Perri D, et al. Naturopathic care for anxiety: A randomized controlled trial ISRCTN78958974. PLoS One 2009;4 (no pagination)(e6628).

29. Seely D, Szczurko O, Cooley K, et al. Naturopathic medicine for the prevention of cardiovascular disease: A randomized clinical trial. CMAJ 2013;185:E409-E416.

30. Beer AM, Ostermann T, Matthiessen PF. Evaluation of inhospital treatment with naturopathic methods - The 'blankenstein model'. Part I: Patients and therapeutic concepts. Forsch Komplementarmed Klass Naturheilkd 2001; 8:6-13.

31. Teut M, Schnabel K, Baur R, et al. Effects and feasibility of an Integrative Medicine program for geriatric patients- $A$ cluster-randomized pilot study. Clin Interv Aging 2013;8: 953-961.

32. Weidenhammer W, Wessel A, Melchart D. Quality profile of a CAM rehabilitation clinic - Part 2: Patients and outcome profile. Forsch Komplementarmed 2007;14:335345 .

33. Wiebelitz KR, Teske W, Henke T, et al. [Naturopathic and orthopaedic in-patient treatment of chronic back pain-a comparison study]. [German]. MMW Fortschr Med 2011; 153 Suppl 2:47-55.

34. Ostermann T, Beer AM, Matthiessen PF. Evaluation of inpatient naturopathic treatment - The Blankenstein model. Part II: Effect size and health status of patients in the course of time. Forsch Komplementarmed Klass Naturheilkd 2002;9:269-276.

35. Beer AM, Ostermann T, Matthiessen PF. Changed quality of life by in-patient naturopathic treatment results of the "Blankenstein Model". [German]. Gesundheitswesen 2001;63:242-247.

36. Bairy S, Kumar AMV, Raju M, et al. Is adjunctive naturopathy associated with improved glycaemic control and a reduction in need for medications among type 2 Diabetes patients? A prospective cohort study from India. BMC Complement Altern Med 2016;16:290-290.

37. Edla SR, Kumar AMV, Srinivas B, et al. 'Integrated Naturopathy and Yoga' reduces blood pressure and the need for medications among a cohort of hypertensive patients in South India: 3-months follow-up study. Adv Integr Med 2016;3:90-97.

38. Murthy SN, Rao NSN, Nandkumar B, Kadam A. Role of naturopathy and yoga treatment in the management of hypertension. Complement Ther Clin Pract 2011;17: 9-12.

39. Rao Y, Kadam A, Jagannathan A, et al. Efficacy of naturopathy and yoga in bronchial asthma. Indian $\mathrm{J}$ Physiol Pharmacol 2014;58:232-238.

40. Sathyaprabha TN, Murthy H, Murthy BTC. Efficacy of naturopathy and yoga in bronchial asthma-A self controlled matched scientific study. Indian J Physiol Pharmacol 2001;45:80-86. 
41. Pradeep N, Mohanty S, Hyndavi S. Evidence for naturopathic and yogic interventions to augment the effects of art care as an adjuvant therapy-A parallel matched control study. Int J Clin Biomed Res 2016;2:19-23.

42. Sarris J, Gadsden S, Schweitzer I. Naturopathic medicine for treating self-reported depression and anxiety: An observational pilot study of naturalistic practice. Adv Integr Med 2014;1:87-92.

43. Braun L, Stanguts C, Spitzer O, et al. A wellness program for cardiac surgery improves clinical outcomes. Adv Integr Med 2014;1:32-37.

44. Arentz S, Smith CA, Abbott J, et al. Combined Lifestyle and Herbal Medicine in Overweight Women with Polycystic Ovary Syndrome (PCOS): A randomized controlled trial. Phytother Res 2017;31:1330-1340.

45. Isbell B, Carroll S. Clinical outcome research at the University of Westminster Polyclinic. Br Naturopathic J 2007; 24:10.

46. Niwa $Y$, Matsuura H, Murakami M, et al. Evidence that naturopathic therapy including Cordyceps sinensis prolongs survival of patients with hepatocellular carcinoma. Integr Cancer Ther 2013;12:50-68.

47. Gagnier JJ, Moher D, Boon H, et al. Investigating clinical heterogeneity in systematic reviews: A methodologic review of guidance in the literature. BMC Med Res Methodol 2012;12:111.

48. World Naturopathic Federation. World Naturopathic Federation: About Naturopathy. 2018. Online document at: http://worldnaturopathicfederation.org/about-naturopathy, accessed July 17, 2018.

49. Beer AM, Uehleke B, Wiebelitz KR. The history of inpatient care in German departments focussing on natural healing. Evid Based Complement Altern Med 2013;2013: 521879.

50. Nair PM, Nanda A. Naturopathic medicine in India. Focus Altern Complement Ther 2014;19:140-147.

51. Hausser T. Update on Naturopathy in Europe: From its roots to global modern practice. Naturopathic Doctor News Rev 2018;14:15-16.
52. Ostermann T, Beer AM, Langhorst J. The effect of integrative in-patient treatment on patients' quality of life: A meta-analysis. J Altern Complement Med 2013;20: A115.

53. Romeyke T, Stummer H. A study of costs and length of stay of inpatient naturopathy-Evidence from Germany. Complement Ther Clin Pract 2011;17:90-95.

54. Loyd I, Hausser T, Wardle J, et al. World Naturopathic Federation Report: Findings from the 1st World Naturopathic Federation survey. Toronto, World Naturopathic Federation, 2015.

55. Hausser T, Loyd I, Yánez J, et al. WNF Naturopathic Roots Report. Toronto, World Naturopathic Federation, 2016.

56. Sur RL, Dahm P. History of evidence-based medicine. Indian J Urol 2011;27:487.

57. Garrow JS. How much of orthodox medicine is evidence based? Br Med J 2007;335:951.

58. Murad MH, Sultan S, Haffar S, Bazerbachi F. Methodological quality and synthesis of case series and case reports. BMJ Evid Based Med 2018;23:60-63.

59. Dwan K, Gamble C, Williamson PR, et al. Systematic review of the empirical evidence of study publication bias and outcome reporting bias-an updated review. PLoS One 2013;8:e66844.

60. Steel A, Goldenberg J, Cooley K. Establishing an international research collaborative for naturopathy: The International Research Consortium of Naturopathic Academic Clinics (IRCNAC). Adv Integr Med 2017;4:93-97.

Address correspondence to: Stephen P. Myers, BNat (Hons) National Centre for Naturopathic Medicine Southern Cross University Lismore 2480 Australia

E-mail: stephen.myers@scu.edu.au 